\title{
DOES SUCCESS ENTAIL ABILITY?
}

\author{
David Boylan* \\ Texas Tech University
}

Imagine a great wave is rising and I have dashed into the sea with my surfboard. You know nothing about me: perhaps I am one of the world's great surfers; perhaps I am a fool. What would it tell you about my abilities, if I were ride into the coast on that wave? Would it mean I am able to surf a wave like that? Does success entail ability?

Call the principle that it does Success. ${ }^{1}$ When we focus on successful action, Success is compelling. Austin (1961) said that "it follows merely from the premise that [a golfer sinks a putt], that he has the ability to do it, according to ordinary English". And indeed when someone succeeds in something, like sinking a putt or surfing a wave, one is forced to concede they were able to do that. This is what Success would lead us to expect.

But when success is not yet assured, the lesson seems different. When said before the fact, the claim that I can surf that wave is strong — it says that surfing that wave is within my control. This intuition, call it the control intuition, drives against Success. Just doing something does not demonstrate it is within my control: flukes do happen. So, if the control intuition is right, success should not demonstrate ability.

*Thanks to the participants of the Rutgers Language Workshop, and Fabrizio Cariani, Justin Khoo, Jeff King, Annina Loets, Matthew Mandelkern, Milo Phillips-Brown, Robert Stalnaker, and Barbara Vetter. Special thanks to Ginger Schultheis for comments on multiple drafts.

${ }^{1}$ I will focus exclusively on whether Success is valid for specific ability attributions which concern whether an agent is able to perform an action at a particular time (generally in the future), like lifting 200 pounds right now or hitting a bullseye on this throw. These are to be contrasted with general or generic ability ascriptions, which say that an agent has the ability to perform an action, not at a specific time, but rather in general. Mandelkern et al. (2017) argue that the semantics for generic ability ascriptions is derived by simply embedding a specific ability ascription under a generic operator. 
To resolve this tension, I connect abilities to the Aristotelian notion that while the past is settled, the future is open. What one is able to do, on my account, depends on what will happen, if one performs certain actions; and what will happen, various philosophers and semanticists have argued, depends on what is settled and what is open. This connection to the open future yields a plausible diagnosis of Success: on my account, success does not strictly speaking entail ability; but other closely related principles are valid.

Here is the plan for the paper. First I try to make the above tension precise. I argue that the appeal of Success is connected to two plausible and related principles: that past success entails past ability, which I call Past Success; and that cannot seems to entail will not, which I call Can't-entails-won't. But, on the other, I show that the control intuition gives rise to counterexamples to Success in cases of inexact ability discussed by Kenny (1976). This tension runs deep: no existing account of abilities, not even those developed in response to Kenny, makes sense of all three points together.

To explain these data, I maintain we must connect the truth of ability claims to the facts about what our options settle and what they leave open, in the sense familiar from the literature on future contingents. I do this within a kind of conditional analysis of ability ascriptions. I first define an operator $\mathcal{W}$ with features attributed to 'will' in the literature on future contingents. In particular, $\mathcal{W} \phi$ is indeterminate in truth-value, when $\phi$ is unsettled. Building on previous work in Mandelkern et al. (2017), I state my conditional analysis in terms of $\mathcal{W}$-conditionals: on my view, 'S can $\phi$ ' says, roughly, there's some action available to $\mathrm{S}$ such that if $\mathrm{S}$ does it, then $\mathcal{W}(\mathrm{S} \phi)$ 's is true. By thus building a connection between unsettledness and indeterminacy into ability claims, my conditional account of abilities reconciles the motivations for Success with its counterexamples.

\section{The Status of Success}

Ability modals are, unsurprisingly, most naturally expressed in English with 'is able to':

(1) I am able to surf that wave. 
They can also be naturally expressed with the modal 'can': I can surf that wave.

Success is a principle governing the logic of ability modals. Formally put, it says:

Success. $\mathrm{S} \phi \models \mathrm{S}$ can $\phi$

Both in Success and later on in my semantics, I focus exclusively on the ability reading of 'can'; I set aside circumstantial, epistemic and deontic uses. ${ }^{2}$

\footnotetext{
${ }^{2}$ There is actually a somewhat subtle question here about what kind of expression ${ }^{\top} \mathrm{S} \phi{ }^{\top}$ is in the statement of Success. It is crucial that ' $\mathrm{S} \phi{ }^{\urcorner}$be the modal's prejacent. But it is generally thought that root modals, such as ability modals, are syntactically below tense. (See Cinque (1999) and Hacquard (2006).) This means that ability modals combine with tenseless phrases and, as a result, strictly speaking the premise of Success is a tenseless phrase that expresses a proposition. (Though note that this does not mean that ${ }^{\mathrm{S}} \mathrm{S} \phi$ ' does not make any reference to times; not all temporal reference is through tense.) This is why I have deliberately omitted any tense marking on ${ }^{\top} \mathrm{S} \phi$ '.

Tenseless phrases are not grammatical sentences of English; they are grammatical subsentential expressions. But they often have the same semantic value of grammatical tensed sentences of English; and so to assess instances of Success and my other principles we will instead assess those sentential paraphrases. Consider the sentence:
}

(i) He can hit the dartboard on the next shot.

Here the prejacent of the modal is a tenseless phrase like:

(ii) He hit the dartboard on the next shot.

I assume (at least until the end of §1.2) that this tenseless phrase expresses the same proposition as the tensed sentence:

(iii) He will hit the dartboard on the next shot.

So to assess the instance of Success:

He hit the dartboard on the next shot $=$ He can hit the dartboard on the next shot.

we can consider whether it is possible for (i) to be false (or not true) while (iii) is true. More generally, the methodology throughout will be to assess the relevant principles by finding grammatical tensed analogues of the premises and conclusions, where necessary. Thanks to an anonymous reviewer for pushing me to clarify this issue. 


\subsection{The Appeal of Success}

Two facts are easy to explain, if Success is valid and hard to explain otherwise. To appreciate the first, let's focus on relatively mundane cases inspired by Kenny (1976)'s discussion of abilities:

Fluky Dartboard. I am a terrible dartplayer. I struggle to even hit the board whenever I take a shot. However, I take my shot and I flukily hit the bullseye.

Once I have taken the shot and hit the bullseye, I can compellingly argue:

I hit the bullseye on that throw.

So, I was able to hit the bullseye on that throw.

If you know that I have been successful, you must concede I was able to. ${ }^{3}$

This is our first point in favour of Success: past success feels like it entails past ability. Where $\leadsto$ denotes a felt entailment, we have: ${ }^{4}$

Past Success. PAST $(\mathrm{S} \phi) \leadsto P A S T(\mathrm{~S}$ can $\phi)$

This seems like strong motivation for Success itself. How could Past Success be valid, if Success is not? After all, I haven't changed since I hit the bullseye. My abilities are what they were some moments ago.

The second fact is that it sounds incoherent to affirm or leave open the premise of Success, but deny the conclusion. Consider:

(4) \# I can't hit the bullseye on this shot, but I will.

${ }^{3}$ Relatedly, Maier (2018) observes that, in cases like Fluky Dartboard it is hard to say whether the agent has the ability to hit a bullseye. I think Maier's observation is well explained in terms of mine: when asked whether I have the ability we are torn between two considerations: there is true, past specific reading that says I was able to hit the dartboard; but there is also a generic reading on which it is not true that I am able to hit the bullseye.

However, my problem goes beyond Maier's observation and cannot be solved using the generic/specific distinction. My observations strongly support that Past Success, the failure of $\vee$ Success and Can't-Entails-Won't all apply to specific abilities: all of my examples use ability ascriptions with a specific prejacent. And so long as we can isolate a single type of reading that all these principles apply to, we will have the problems identified in $\$ 1.3$.

${ }^{4}$ This is something like the converse of the actuality entailments discussed by Bhatt (1999) and Hacquard (2006). 
As I invite you to verify, in general sentences of the form ' $\mathrm{S}$ can’t $\phi$ but will $\phi\urcorner$ sound absurd.

This is our second point in favour of Success. In general, it seems that can't entails won't:

Can't-entails-won't. $\neg(\mathrm{S}$ can $\phi) \leadsto \neg(\mathrm{S} \phi)$

And Can't-entails-won't is just the contrapositive of Success.

\subsection{A Counterexample to Success}

That is the case for Success, as I see it. But at the end of the day, I think it cannot be valid. Recall the control intuition: if I say something like

(6) I can hit the bullseye on this throw.

I say something quite strong. (6) is not verified by the small chance of me hitting the dartboard.

We can leverage this intuition to find counterexamples to Success. Take the following variation on the dartboard case inspired by Kenny (1976): ${ }^{5}$

Unreliable Dartboard. I am a fairly bad dartplayer. I regularly hit the bottom half when I aim for the top; and vice versa. But I never miss the board entirely.

I am about to take a shot. I am skilled enough to know I will hit the board; so I know the following:

(7) I will hit the top half of the board on the next shot or I will hit the bottom half of the board on this throw.

\footnotetext{
${ }^{5}$ This argument is closely related to Kenny's point that ability modals fail to distribute across disjunction. I am indebted to Fusco (2021) here, which directed my attention specifically to $\vee$-Success (though her focus is not the status of Success). However, see the end of $\$ 1.3$ for discussion of how my puzzle goes beyond Kenny's point.
} 
But it does not seem that I should ascribe myself either of the following abilities here:

(8) I can hit the top on the throw.

(9) I can hit the bottom on this throw.

Even the disjunction does not seem true:

(10) I can hit the top of the board on this throw or I can hit the bottom of the board on this throw.

So, in advance of the shot, even if I know I will hit the board somewhere, I do not have the ability to hit the top or the ability to hit the bottom.

But I would be predicted to have one ability or the other if Success were valid. For I will hit either the top or the bottom of the dartboard. In the first case, Success says I'm able to hit the top, in the second that I'm able to hit the bottom. In either case (10) should be true, if Success is valid. But it is not.

Here is that argument again, with all the steps made explicit:

1. I will hit the top half of the board on the next shot or I will hit the bottom half of the board on this throw.

(Premise)

2. I will hit the top half of the board on the next shot. (Assumption for Conditional Proof)

3. I can hit the top half of the board on the next shot. (Success, (2))

4. I will hit the top half of the board on the next shot $\supset$ I can hit the top half of the board on the next shot. (Conditional Proof, (2), (3))

5. I will hit the bottom half of the board on the next shot. (Assumption for Conditional Proof)

6. I can hit the bottom half of the board on the next shot. $\quad$ (Success,(5))

7. I will hit the bottom half of the board on the next shot $\supset$ I can hit the bottom half of the board on the next shot. (Conditional Proof, (5), (6)) 
8. I can hit the top half of the board on the next shot or I can hit the bottom half of the board on the next shot. $\quad$ (Constructive Dilemma, (1), (4), (7))

All steps but two appeal to highly plausible principles of classical logic; the clear weak links are the appeals to Success at steps (3) and (6). ${ }^{6}$

The consequences of Success become more absurd as the disjunctions get longer. Suppose we divide the dartboard into a million tiny, numbered regions. I can see that I will hit (at least) one of these regions because I know I will hit the dartboard, yielding:

$$
\text { I will hit region } 1 \text { or } 2 \text { or } 3 \text { or ... or } 1,000,000 \text { on this throw. }
$$

If Success is valid, then the following incredible claim is true:

I can hit region 1 on this throw or I can hit region 2 on this throw or ... or I can hit region $1,000,000$ on this throw.

And, to put an even finer point on it, notice that each disjunct of (12) entails: ${ }^{7}$

There is a certain point that I can hit on this throw.

Success predicts that (13) should just be a truism here. In fact, (13) would be an incredible boast for me to make in Unreliable Dartboard.

So you can be sure that you will make true some disjunction, while failing to have the ability to make true either disjunct. This gives us the failure of the following inference pattern:

$\vee$ - Success. $\mathbf{S} \phi \vee \mathrm{S} \psi \rightsquigarrow \mathrm{S} \operatorname{can} \phi \vee \mathrm{S} \operatorname{can} \psi$

And, as we saw, $\vee$-Success is a consequence of Success, given natural classical forms of reasoning.

\footnotetext{
${ }^{6}$ The other classical principles are not completely beyond reproach. Informational accounts of logic, as defended in Yalcin (2007) and Bledin (2014), deny Constructive Dilemma. However, Constructive Dilemma will be valid even on informational approaches when restricted to premises not containing epistemic vocabulary like epistemic modals or conditionals; so even informational accounts should deny Success here.

${ }^{7}$ Here I use 'certain' to single out the reading where the quantifier takes wide scope.
} 
One might worry about the tense of the counterexample. ${ }^{8}$ Success and $\vee$ Success are principles governing your present abilities; but my example concerns the future. How then could Unreliable Dartboard have the form of a counterexample to $\vee$-Success?

Consider first the conclusion of $\vee$-Success. This is indeed in the present tense. But so too are the ability modals in (10). We must distinguish between the tense of the ability and the time of the embedded action. Consider:

I am able to hit the dartboard on the next shot.

The claim is in the present tense: it is about what I am able to do. Nonetheless this (present) ability claim concerns future actions: the action hit the dartboard on the next shot clearly refers to the future. We cannot infer that an ability claim is not present tensed, simply because it concerns actions in the future. So $\vee$-Success, though a principle about one's present abilities, still governs (present) abilities to do future actions, like hitting the dartboard on the next shot. Future actions are a legitimate source of counterexamples to the principle.

What about the premise of $\vee$-Success? In my example, the premise is not in the simple present, since it is about the future. ${ }^{9}$ But $\vee$-Success does not demand that the premise be in the present tense. It simply requires that the $\phi$ 's and $\psi$ 's embedded under the modals be the same as those in the premises. If in a given instance of $\vee$-Success the $\phi$ 's and $\psi$ 's are future oriented, then the premise must be too. But a future oriented (non-modal) premise cannot be in the simple present; so the premise of that instance of $\vee$-Success cannot be in the present. This is exactly the case in Unreliable Dartboard and so the future directedness of (7) does not render the example illegitimate.

But perhaps (7) cannot be the premise of an instance of $\vee$-Success, even granting the point about future directedness. The prejacents of 'can' in the conclusion are something like:

I hit the top of the dartboard on the next shot.

\footnotetext{
${ }^{8}$ Thanks to an anonymous referee for pushing me to consider this worry.

${ }^{9}$ Strictly speaking, it is in the present tense, as English does not have a future tense, but rather the present-tensed but future-shifting auxiliary 'will'. So I say instead it is not in the simple present.
} 
I hit the bottom of the dartboard on the next shot.

These are not the disjuncts in (7). ${ }^{10}$ Those contain an extra auxiliary, namely will:

(17) I will hit the top of the dartboard on the next shot.

(18) I will hit the bottom of the dartboard on the next shot.

So we might still worry that the $\phi$ 's and $\psi$ 's in my example are not uniformly instantiated in the premises and conclusion; they contain extra material.

Many accounts of 'will' make no semantic distinction between (15) and (16) on the one hand and (17) and (18) on the other. If, for instance, 'will' is just a forward shifting temporal operator, then it is a redundant addition to (17) and (18). ${ }^{11}$ So, on such an accounts, the above pairs are equivalent and the counterexample goes through: if either of (17) or (18) is true, then so must one of (15) and (16).

But there is an important class of views that denies the above pairs are equivalent. Some modal accounts of 'will', such as those explored by Prior (1967) and Belnap et al. (2001) say that ${ }^{\ulcorner} \mathrm{Will} \phi{ }^{\top}$ is true iff $\phi$ is settled. Settledness is a stronger notion than actual future truth; so modal theorists will distinguish between (15) and (16), on the one hand, and (17) and (18) on the other. One might conclude then that modal theories offer an escape from the counterexample: the variables in $\vee$-Success are not uniformly nor even equivalently instantiated.

Not so. These modal approaches reject the wrong half of the equivalence, at least for the purposes of evading my counterexample. They say (15) and (16) do not entail (17) and (18):

$$
\mathrm{S} \phi \text { at } t^{\prime} \not=\mathrm{Will}\left(\mathrm{S} \phi \text { at } t^{\prime}\right)
$$

But, as any plausible view of 'will' should, they accept the converse: ${ }^{12}$

\footnotetext{
${ }^{10}$ As I discuss in the earlier footnote $2,(15)$ and (16) are really tenseless phrases.

${ }^{11}$ The same goes for Cariani and Santorio (2018)'s selection function semantics for 'will'.

${ }^{12}$ As noted by Ninan (2021), there are some modal views, particularly in the linguistics literature, which in fact deny this entailment. For instance, Copley (2009) takes 'will' to universally quantify over normal futures; but since the actual future might be abnormal (20) will fail on her account. But, as Ninan convincingly argues, this is a substantial problem for such views: 'will'-claims will sometimes be true, even if their prejacent turns out to be false, so long as the actual future is not a normal one. Some further independent evidence for this: if (20) fails, sentences like the following
} 


$$
\text { Will (S } \left.\phi \text { at } t^{\prime}\right) \models \mathrm{S} \phi \text { at } t^{\prime}
$$

After all, settledness entails truth.

This is sufficient to reconstruct the counterexample. We saw in Unreliable Dartboard that (7) is true: ${ }^{13}$

(7) Either I will hit the top half of the board on the next shot or I will hit the bottom half of the board on the next shot.

(7) and Constructive Dilemma allow us to derive the disjunction:

(21) I hit the top half of the board on this throw or I hit the bottom half of the board on this throw.

(21) and (10) together supply the counterexample to $\vee$-Success, one where we can now be sure that the $\phi$ 's and $\psi$ 's are instantiated univocally. ${ }^{14}$

\subsection{Deepening the Tension}

We now have three pieces of data:

can be coherent:

(i) \#It will rain tomorrow but it might not rain tomorrow.

For this reason, I constrain my attention to modal views that do validate the above entailment.

${ }^{13}$ The modal theorist's last resort here might be to deny (7) and claim only the following wide-scope will-claim is true:

(i) I will hit the top or the bottom of the dartboard.

This is in fact a prediction of their semantics, as they deny Will Excluded Middle:

Will Excluded Middle. Will $\phi \vee$ Will $\neg \phi$

The plausibility of this prediction is questionable - my truth-value judgements do not distinguish between (7) and (i). But more importantly, this move still does not defuse the counterexample. Given the principle (20), we can derive the 'will'-free (21) directly from (i); the reasoning proceeds the same from there.

${ }^{14}$ Going forward I will take the more natural (7) and (10) to constitute the counterexample; the inclined reader may substitute (21) for (7) throughout. 
Validity of Past Success. PAST (S $\phi) \leadsto P A S T(\mathrm{~S}$ can $\phi)$

Validity of Can't-entails-won't. $\neg(\mathrm{S}$ can $\phi) \leadsto \neg(\mathrm{S} \phi)$

Invalidity of $\vee$-Success. $\mathbf{S} \phi \vee \mathrm{S} \psi \rightsquigarrow \mathrm{S} \operatorname{can} \phi \vee \mathrm{S} \operatorname{can} \psi$

Predicting this combination is a serious challenge. I show Past Success and Can'tentails-won't are equivalent to Success given standard assumptions. I will focus on a particular case study, a standard modal analysis of abilities, for illustration. But the argument generalises: no existing account has the resources to make sense of all three of our principles. ${ }^{15,16}$

The modal analysis of ability ascriptions, defended by Hilpinen (1969), Lewis (1976), Kratzer (1977) and Kratzer (1981), says that 'can' is a standard possibility modal, like 'might' or 'is possible': 'S can $\phi$ ' is true iff, within some set of accessible worlds, there is some possible world where $\mathrm{S} \phi$ 's. The accessible worlds are generally taken to be circumstantially possible worlds that hold fixed the intrinsic properties of the agent. More precisely, where $\mathbb{A}(w)$ is the set of accessible worlds at $w:^{17}$

Modal Analysis. $\llbracket$ can $(\mathrm{S} \phi) \rrbracket^{w}=1$ iff $\exists w^{\prime} \in \mathbb{A}(w): \llbracket \mathrm{S} \phi \rrbracket^{w^{\prime}}=1$.

\footnotetext{
${ }^{15}$ An anonymous referee notes an interesting and similar puzzle regarding estimations of skill. In Fluky Dartboard it initially seems right to say that I am a terrible dartplayer. But if I go on to then hit the dartboard, this seems to put pressure on my claim in retrospect.

Unlike my puzzle, I think this one should be solved epistemically. There is indeed a tendency to infer that I do not (determinately) have the ability to make the dartboard on this shot, if I am a terrible dartplayer. But this inference is merely defeasible - it is coherent to say things like:

He is a terrible dartplayer, though I admit he was able to hit the dartboard on that shot.

Nonetheless, as is typical even of defeasible inferences, my hitting the board is generally some evidence that I was able to do so; and so it is some evidence against the claim that I am terrible dartplayer. Unless my audience has good independent information that I am a terrible dartplayer, my actually hitting the dartboard should make them less confident of my claim. This I think gives a good account of the pressure put on my claim by actual success: it tends to force me to give new evidence for my claim.

${ }^{16}$ Willer (2021)'s system does predict the simultaneous failure of Success and the validity of Can'tentails-won't. It does not however predict the validity of Past Success.

${ }^{17}$ Here and throughout I make the background assumption that, at LF, 'can' is really a sentential operator, taking a sentence as argument; and not a raising modal with a more complicated syntax. This is simply to aid presentation (and the proofs in the Appendix).
} 
No matter what worlds are accessible, this view cannot accommodate both the invalidity of Success and the validity of Can't-entails-won't. Can't-entails-won't is valid iff $\mathbb{A}$ is reflexive, i.e. $\mathbb{A}(w)$ always contains $w$. But reflexivity guarantees Success is valid too. This is exactly because Can't-entails-won't is just the contrapositive of Success.

Now turn to the second tension, that Past Success is valid while Success is not. Most accounts are not explicit about the role of time in ability modals. ${ }^{18}$ But the natural way of adding temporal sensitivity to these accounts does not resolve the tension between Success and Past Success. Take an operator view of past tense, where ${ }^{r} P A S T \phi '$ is true at a time $t$ iff $\phi$ was true at some previous time $t:{ }^{19}$

$$
\llbracket P A S T \phi \rrbracket^{w, t}=1 \text { iff } \exists t^{\prime}<t: \llbracket \phi \rrbracket^{w, t^{\prime}}=1
$$

Remember, the modal analysis says that ${ }^{\ulcorner} \mathrm{S}$ can $\phi^{\top}$ is true at $w$ iff there is some accessible world where S $\phi$ 's. But one's abilities are not fixed once and for all: people lose abilities and gain new ones too. The natural fix is to say that what worlds are accessible changes over time. ${ }^{\mathrm{r}} \mathrm{S}$ can $\phi{ }^{\urcorner}$is true at a world $w$ and a time $t$ iff there is some world accessible from $w$ at $t$ where $\mathbf{S} \phi$ 's. Where $\mathbb{A}(w)(t)$ is the set of worlds accessible at $w$ and at time $t$, we now have:

$$
\llbracket \operatorname{can}(\mathrm{S} \phi) \rrbracket^{w, t}=1 \text { iff } \exists w^{\prime} \in \mathbb{A}(w)(t): \llbracket \mathrm{S} \phi \rrbracket^{w^{\prime}, t}=1 .
$$

Now we can see why Past Success is invalid iff Success is. Suppose, on Monday, I hit the dartboard even though I am not able to, invalidating Success. Then on Tuesday it will be true that, on Monday, I hit the top of the dartboard; but it will also be true on Tuesday that, on Monday, I wasn't able to hit the top of the dartboard, invalidating Past Success as well. The argument that Success is valid, if Past Success is, proceeds the same way: a counterexample to Past Success at $t$ will be a counterexample to Success at some point earlier than $t$.

The modal view is just one account of abilities; but the problems it faces are general. Before going on, it's worth considering one more class of views, defended by Brown (1988), Horty and Belnap (1995) and Horty (2001). ${ }^{20}$ Such views say

\footnotetext{
${ }^{18}$ An exception to this is Horty (2001). But that view does not escape the challenge here.

${ }^{19}$ The point goes through equally well on a referential theory of the past tense.

${ }^{20}$ A related view is the conditional analysis of Mandelkern et al. (2017), where ${ }^{\ulcorner} \mathrm{S}$ can $\phi^{\urcorner}$is true just
} 
that $\left.{ }^{\top} \mathrm{S} \operatorname{can} \phi\right\urcorner$ is true iff there's some proposition in the agent's power to bring about that entails S $\phi$ 's; I'll call this the boxy view. More formally, where $\mathcal{A}(w)$ is a set of accessible propositions at $w$, the boxy view says:

Boxy Analysis. $\llbracket \operatorname{can}(\mathrm{S} \phi) \rrbracket^{w}=1$ iff $\exists p \in \mathcal{A}(w): \forall w^{\prime} \in p: \llbracket \mathrm{S} \phi \rrbracket^{w^{\prime}}=1$.

This view does no better: Success, on this view, is just equivalent to Can't-entailswon't; both are characterised by the condition that $\{w\} \in \mathcal{A}(w) .{ }^{21}$ And, for the same reasons as on the modal analysis, the natural way of adding time to the analysis validates Past Success iff it validates Success.

This point is worth dwelling on for a moment longer. The boxy analysis has been proposed (at least in part) to specifically account for Kenny (1976)'s observation that ability does not distribute across disjunction. The fact that it fails demonstrates that my puzzle here goes beyond Kenny's: even accounts that do not predict distribution over disjunction fail to account for my three data points.

\section{The Path Forward: the Indeterminacy View}

There is a tight connection between what we want and what we do not. What kind of resources would allow us to pull these principles apart?

in case for some available action if $\mathrm{S}$ were to perform that action, $\mathrm{S}$ would $\phi$; the relevant conditional here is the Stalnakerian variably strict conditional, which obeys Conditional Excluded Middle.

To spell this out more formally, let $\mathcal{A}(w)$ the agent's available actions at $w$. Write $f(\mathbf{A}, w)$ for the closest world where A holds. Then the Mandelkern et al. semantics is as follows:

$$
\llbracket \operatorname{can}(\mathbf{S} \phi) \rrbracket^{w}=1 \text { iff for some } \alpha \in \mathcal{A}(w): f(\mathbf{S} \text { tries to } \alpha, w) \in \llbracket \mathrm{S} \phi \rrbracket
$$

This analysis, while close to my own, faces the same problems as the views above. Success and Can't-entails-won't are both characterised by the condition that the agent always tries to perfrom some available action:

(i) For some $\alpha \in \mathcal{A}(w), w \in \mathbf{S}$ tries to $\alpha$.

I show in Appendix A that this characterises Success in their framework; the reader can easily adapt the proof to show that it also characterises Can't-entails-won't. Moreover, once times are added to the analysis, Past Success is equivalent Success for just the same reasons as on the modal analysis: a counterexample to Success at $t$ is a counterexample to Past Success at some time later than $t$; and vice versa.

${ }^{21}$ See Appendix A for the proof. 
Start with Can't-entails-won't and $\vee$-Success. Here we want to say that one inference pattern, Success, is invalid, but its contrapositive, Can't-entails-won't, is not. If we say sentences can be neither true nor false, but rather indeterminate, this allows for exactly the structure we want. If entailment is truth-preservation, the inference from $\phi$ to $\psi$ can be invalid, because $\psi$ can be indeterminate when $\phi$ is true, even while the inference from $\neg \psi$ to $\neg \phi$ is in fact valid.

To see this, focus on a toy case. Suppose we have three worlds $w_{1}, w_{2}$ and $w_{3}$ and two sentences $\phi$ and $\psi$. Writing '\#' for indeterminacy, suppose that $\phi$ and $\psi$ have the values shown below:

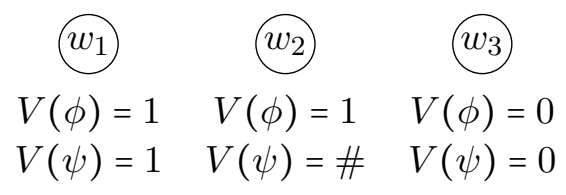

Assume entailment is truth-preservation. Then $\phi$ does not entail $\psi$ : there is a world where $\phi$ is true but $\psi$ is not, namely $w_{2}$; at $w_{2}$ it is not true but indeterminate. But $\neg \psi$ does entail $\neg \phi$ : at every world where $\neg \phi$ is true, namely $w_{3}, \neg \phi$ is also true. This, I suggest, is exactly the situation with Success and Can't-entails-won't. Can't-entails-won't always preserves truth; Success does not, because sometimes its premise is true, while its conclusion is indeterminate.

Now take Past Success and Success. Reasoning according to Past Success, but not Success, is good, I'll suggest, because when success is in the past, it is settled. Since Aristotle at least, it has been recognised that there is some sense in which the future is open, but the past is settled. Suppose when I tossed a fair coin once it came up heads and I am about to toss it again. The outcome of the first toss is settled: the course of history so far determines that it came up heads. The outcome of the second is unsettled: history does not settle how it will land, since it is fair.

But in what sense is the future open and the past settled? Perhaps outcomes can be metaphysically indeterminate, as, for example, Barnes and Cameron (2011) argue: perhaps all the facts up to now plus the laws of nature really do not settle certain outcomes. But this is a controversial metaphysical position. I aim to be more ecumenical. I take the outcome of the coin flip to be unsettled in the sense that the coin could, in the everyday sense, come up heads or come up tails. Following 
Condoravdi (2002), I take this everyday sense of possibility to be what Kratzer (1981, 1991) calls circumstantial possibility. It is plausibly understood as holding fixed just some relevant subset of facts up until now. Perhaps the microlevel facts and the laws settle what will happen with the coins, perhaps they don't. But holding fixed facts like, say, the coin is two sided and on average it comes up heads half the time does leave open whether it will come up heads or not.

These notions have of course been studied together extensively in the literature on 'will' and future contingents. So that is where I will look for inspiration for my account of abilities.

\section{Future Contingents and $\mathcal{W}$}

Future contingents have been argued to have three special properties. As we will see, each property mirrors a property of ability. Our assessments of 'will'-claims, it has been argued, involve a kind of temporal asymmetry. Before the fact, the future seems open and 'will'-claims seem unsettled; but after the fact, we seem happy to talk as if they were settled all along. 'Will' is also scopeless with respect to negation: ${ }^{\ulcorner} \neg$ Will $\phi$ ' seems equivalent to ${ }^{\ulcorner}$Will $\neg \phi{ }^{\top}$.

Here I introduce a modal operator $\mathcal{W}$ that captures this behaviour. $\mathcal{W}$ is a selection modal, in the sense of Cariani and Santorio (2018): ' $\mathcal{W} \phi{ }^{\urcorner}$says that $\phi$ is true in the closest world to actuality. But crucially, on my theory, the closest world leaves various facts unsettled. This ensures $\mathcal{W}$ has the right properties for giving the semantics of ability modals.

\subsection{Features of Future Contingents}

Let's first take the temporal asymmetry in assessing future contingents.

Recall Aristotle's famous case of the sea battle. On Monday, it is not yet settled whether there will be a battle or not on Tuesday: a capricious ruler decides by flipping a fair coin this evening. I make the following prediction:

There will be a sea battle on Tuesday.

There is a long tradition of thinking that because the future is unsettled, sentences 
like (24) must be indeterminate in truth-value. ${ }^{22}$ Things could go either way, depending on how the coin lands. If the coin comes up heads, there will be a battle; if not then not. But the outcome of the toss is not settled; and so whether there will be a sea battle tomorrow is unsettled too.

Now suppose the sea battle does take place on Tuesday. When I look back on my earlier prediction, what should I think? It seems I can say either of the following:

There would in fact be a sea battle on Tuesday.

(26) I said there would be a sea battle on Tuesday; and indeed there would be.

'Would' is generally regarded to be the past tense of 'will'. ${ }^{23}$ But then it is surprising that we can say either of (25) or (26): if my assertion was indeterminate when I said it, why do I now say that it was true ${ }^{24}$

These are the first two properties I want $\mathcal{W}$ to have. Where $\diamond \phi$ says that $\phi$ is circumstantially possible and $\nabla \phi$ says that $\phi$ is indeterminate, we have:

Openness. $\diamond \phi, \diamond \neg \phi \leadsto \nabla \mathcal{W} \phi$

Past Settledness. PAST $\phi \leadsto$ PAST $\mathcal{W} \phi$

Before the fact, if $\phi$ and $\neg \phi$ are possible, then $\mathcal{W} \phi$ is indeterminate; but once $\phi$ has happened, PAST $\mathcal{W} \phi$ is determinately true. (As we will see later on, this will eventually allow me to validate Past Success without validating Success.)

The third feature is the way that 'will' interacts with negation. Usually, placing a negation over or under a modal gives rise to very different meanings. Saying something like

(27) You have to not be there when he arrives.

means something very different from

(28) You don't have to be there when he arrives.

\footnotetext{
${ }^{22}$ See, among others, Aristotle, De Interpretatione, Lukasiewicz (1920), Lukasiewicz (1951).

${ }^{23}$ See Abusch (1997), Abusch (1998) and Condoravdi (2002).

${ }^{24}$ See MacFarlane (2003).
} 
This scoping behaviour is typical of modals. In general:

$$
\neg(M O D A L \phi) \not=M O D A L \neg \phi
$$

'Will' does not give rise to any scope distinctions with respect to negation. Take a predicate like 'is absent' that includes a negation as part of its meaning. Consider the following example:

I doubt that John will be present.

This says that I think it is not the case that John will be present. But it quite clearly entails

(31) I think that John will be absent.

In general, saying that it is not the case that $\phi$ will happen just is to say that $\neg \phi$ will happen. ${ }^{25}$ I aim then for $\mathcal{W}$ to be scopeless with respect to negation, as Cariani and Santorio (2018) put it:

Scopelessness. $\neg \mathcal{W} \phi \leftrightarrow \mathcal{W} \neg \phi$

(As we'll see later, Scopelessness will secure Can't-entails-won't.)

\subsection{Combining Selection Modals with Indeterminacy}

Following Cariani and Santorio (2018), I say that ${ }^{\ulcorner} \mathcal{W} \phi{ }^{\top}$ is true iff $\phi$ is true in the closest world to the actual world. ${ }^{26}$

But plugging this into the standard way of thinking about worlds does not yield indeterminacy. Worlds in possible worlds semantics are usually treated as complete possible histories: when you give a world a proposition and time, it always tells

\footnotetext{
${ }^{25}$ This point has been recognised at least since Thomason (1970).

${ }^{26}$ An alternative starting point would be a 'Peircian' quantificational view of 'will', where ${ }^{\ulcorner} \mathcal{W} \phi$ ' says that $\phi$ is true in all possible histories compatible with the present. This has been explored by, among others, Prior (1967) and Belnap et al. (2001) and in linguistics by Kaufmann (2005) and Copley (2009). I do not opt for a Peircian view because using it in my semantics for 'can' has unwelcome consequences. We validate a much stronger inference than Past Success, namely the inference from $\left.{ }^{\ulcorner} P A S T \mathrm{~S} \phi\right\urcorner$ to ${ }^{\ulcorner} P A S T \mathrm{~S}$ cannot not $\left.\phi\right\urcorner$. But this inference is clearly invalid.
} 
us that the proposition is either true or false there at that time. So if $\mathcal{W}$ selects a complete possible history, then it does not introduce indeterminacy. ${ }^{27}$

Nevertheless, possible worlds semantics has the resources to represent a world that is unsettled in various respects. As shown by Prior (1967), Thomason (1970) and Thomason (1984), a world that is unsettled past a certain time can be represented using a set of worlds which agree in all (relevant) matters of fact up to that time, but diverge afterwards; I'll call such a set an unsettled world. In the sea battle case, we represent the earlier, indeterminate state of the world with a set of worlds agreeing on all (relevant) matters of fact up until today and then diverging on whether a sea battle occurs tomorrow. We can think of a proposition as being true at an unsettled world iff it is true at all worlds in that set; as false iff it is false at all worlds in that set; and indeterminate, otherwise

I combine the selection modal semantics with unsettled worlds. Say that the actual unsettled world represents our world at the time of utterance, settling everything up until that time. ' $\mathcal{W} \phi{ }^{\top}$ says that $\phi$ is true in the closest unsettled world to the actual unsettled world. Crucially, the closest world to an unsettled world will usually be the actual unsettled world itself, and so various $\mathcal{W}$-claims to be themselves indeterminate.

Before we go on, we should acknowledge an obvious question - is $\mathcal{W}$ the semantics for 'will'? ${ }^{28}$ I want to remain neutral here. All of the properties of my $\mathcal{W}$ have defenders in the literature on 'will', but they are still controversial. Particularly so for Openness: many deny that settledness plays any interesting role in the truth of 'will' claims. ${ }^{29}$ (Indeed, the tradition of rejecting this principle is almost as old as the tradition that embraces it.) This is why I have introduced $\mathcal{W}$ as a separate operator: my core claim is that the kind of indeterminacy that has been attributed by some to 'will' plays a crucial role in ability ascriptions; and not that 'will' itself means $\mathcal{W}$.

\footnotetext{
${ }^{27}$ Cariani and Santorio are aware of this but prefer to keep indeterminacy in the postsemantics.

${ }^{28}$ Thanks to an anonymous referee for pushing me clarify my thoughts here.

${ }^{29}$ Note, however, that it is often accepted in natural language semantics that something like the open future is involved in other constructions. In particular, it is often assumed that circumstantial modality has the property that past and present propositions are either necessarily true or necessarily false; while claims about the future may be both possibly true and possibly false. Condoravdi (2002) puts this property to work in accounting for contrasts between the temporal orientation of circumstantial and epistemic modals.
} 
If 'will' is not sensitive to settledness, then perhaps my use of unsettled worlds and the indeterminacy they generate are sui generis. Or perhaps not. One source of indeterminacy in natural language is the phenomenon of homogeneity presuppositions. It was noted in Fodor (1970) that plurals appear to presuppose that their domain is homogenous with respect to their arguments. Consider:

The boys are in the yard. The boys are not in the yard.

Neither sentence is clearly true, when some but not all the boys are in the yard; both receive truth-values only if the boys are homogenous with respect to whether they are in the yard. Homogeneity has been explored for a range of constructions, modals and conditionals in particular, in Schwarzschild (1993), von Fintel (1997), Kriz (2015), Santorio (2018) and Goldstein (2019); and Copley (2009) proposes it for 'will', but not in a selection modal framework.

The indeterminacy of $\mathcal{W}$ could be reduced to a homogeneity presupposition. We might say that $\mathcal{W}$ presupposes that the closest unsettled world is homogenous with respect to the antecedent. My talk of a proposition being true or false at an unsettled world would simply be a picturesque way of stating the homogeneity constraint; and the key logical features of 'can' would ultimately derive from homogeneity.

A different strategy would be to more thoroughly embrace the idea that propositions are evaluated for truth or falsity at entities coarser than worlds. My unsettled worlds are very close to the possibilities from Humberstone (1981)'s possibility semantics - just like possibilities, they are world-like entities that carry less information than worlds do. (And, like on Humberstone's approach, they are modelled as sets of wholly determinate worlds.) The core idea of possibility semantics is that all sentences are evaluable at possibilities, rather than worlds, ${ }^{30}$ and a key consequence is that, since possibilities do not specify all the facts, sentences can be indeterminate. Stating my semantics in the framework of possibility semantics would thus

\footnotetext{
${ }^{30}$ This is just one of a family of related approaches here. Structurally similar ideas are proposed in the framework of data semantics from Veltman (1985) and its inquisitive semantic descendants, explored in Ciardelli et al. (2018). Truth-maker semantics, as advocated by Fine (2017), also allow for a similar kind of indeterminacy.
} 
reduce the indeterminacy I am interested in to the more general kind deriving from possibilities. I will not offer here a formulation of my semantics in that framework; but I am optimistic it can be done.

I won't take a stand here on which of the three approaches, sui generis unsettled worlds, a homogeneity presupposition, or possibility semantics, is most promising, if 'will' does not mean $\mathcal{W}$; I take all three to be live options. Instead I note that the formal framework below is, in principle, compatible with all three.

\subsection{The Formal Details}

To state the lexical entry for $\mathcal{W}$, let's first say how unsettled worlds get into the semantics and how we model closeness.

I add an unsettled world, $\mathcal{I}$, to the index of the semantic evaluation function $\llbracket \cdot \rrbracket$. I assume that the unsettled world is supplied to the semantics by context: we form the unsettled world of the context $\mathcal{I}_{c}$ by taking the set of worlds that are duplicates of the (determinate) context world up until the context time:

Unsettled World. $\mathcal{I}_{c}=\left\{w \mid w\right.$ is identical to $w_{c}$ up until $\left.t_{c}\right\}$

Now let's think about how to model closeness. Following Stalnaker (1968), I use a selection function to supply the closest worlds. A Stalnakerian selection function $s$ takes a world $w$ and a proposition $\mathbf{A}$ and returns the closest world to $w$ where $\mathbf{A}$ is true. My selection functions take an unsettled world as input and can also return an unsettled world as output: $s(\mathcal{I}, \mathbf{A})$ picks out the closest (possibly unsettled) world to $\mathcal{I}$ which settles that $\mathbf{A}$ is true.

What if we want the selection function to give us the closest world to $\mathcal{I}$ simpliciter? We simply let the other argument be the tautology $T$. I say that $\mathrm{T}$ is supplied by a modal base, a function $f$ from a world and a time to a set of worlds. ${ }^{31}$ I assume that $f$ is supplied by the index and that $f$ does not include any information by itself: in general, $f(w, t)=\mathrm{T}$. To find the closest world simpliciter to the unsettled world of the context, $\mathcal{I}_{c}$, we find $s\left(\mathcal{I}_{c}, f_{c}\left(w_{c}, t_{c}\right)\right)$.

I will make a structural assumption about closeness, which I call Overlap: ${ }^{32}$

\footnotetext{
${ }^{31}$ Cariani and Santorio also use a modal base, but do not assume it is empty.

${ }^{32}$ This is an analogue of the Strong Centering principle on Stalnaker selection functions, which
} 
Overlap. If $\mathbf{A} \cap \mathbf{B} \neq \varnothing$, then $s(\mathbf{A}, \mathbf{B})=\mathbf{A} \cap \mathbf{B}$.

Suppose we want the closest worlds to an unsettled world $\mathbf{B}$ where $\mathbf{A}$ happens and that B contains some A-worlds. Overlap tells us that those closest worlds will be the $\mathbf{A}$-worlds in $\mathbf{B}$.

Overlap guarantees that $\mathcal{I}_{c}$ is the closest world to itself. Since $f_{c}\left(w_{c}, t_{c}\right)$ is the set of all worlds, $s\left(\mathcal{I}_{c}, f_{c}\left(w_{c}, t_{c}\right)\right)$ must be $\mathcal{I}_{c}$. This allows us to ignore the modal base when 'will' is unembedded or under past tense. (Why include it at all then? Because we will use it in the semantics for conditionals: following Kratzer I will assume that conditionals add the antecedent to the modal base.)

Now we can state the semantics. To see if ' $\mathcal{W} \phi{ }^{\top}$ is true, we find $s(\mathcal{I}, f(w, t))$, the closest world to the unsettled world where $\phi$ is true. If $\phi$ is true at $s(\mathcal{I}, f(w, t))$ (i.e. true throughout $\mathcal{I}$ ), ${ }^{r} \mathcal{W} \phi{ }^{\top}$ is true; if $\phi$ is false at $s(\mathcal{I}, f(w, t)$ ) (i.e. false throughout $\mathcal{I}),{ }^{r} \mathcal{W} \phi{ }^{\top}$ is false; but if $\phi$ is neither true nor false at $s(\mathcal{I}, f(w, t)$ ) (i.e. is true at some worlds in $\mathcal{I}$ but false at others), ${ }^{r} \mathcal{W} \phi{ }^{\prime}$ is indeterminate.

Say that the value of $\llbracket \phi \rrbracket$ is determinate at a point just it is not indeterminate. Then we can make this precise as follows: ${ }^{33}$

a. $\llbracket \mathcal{W} \phi \rrbracket^{w, t, f, \mathcal{I}}$ is determinate only if either

(i) $s(\mathcal{I}, f(w, t)) \subseteq \llbracket \phi \rrbracket^{t, f, \mathcal{I}}$ or

(ii) $\quad s(\mathcal{I}, f(w, t)) \subseteq \llbracket \neg \phi \rrbracket^{t, f, \mathcal{I}}$

b. If determinate, $\llbracket \mathcal{W} \phi \rrbracket^{w, t, f, \mathcal{I}}=1$ iff $s(\mathcal{I}, f(w, t)) \subseteq \llbracket \phi \rrbracket^{t, f, \mathcal{I}}$

Finally, some background assumptions. I continue to work with the operator semantics for past tense:

says that $w$ is always the closest world to itself.

${ }^{33}$ Note that, as stated, $\mathcal{W}$ actually includes no time shifting component. For our purposes here, it won't be necessary to add one. We are dealing throughout with specific ability attributions, where the time is specifically built into the prejacent. So, for the relevant 'will' claims, an explicit (future) time will also already be built into the prejacent.

Adding a time-shifting element to the semantics is somewhat delicate: if we simply add existential quantification over future times in the closest world, we no longer validate Scopelessness. One way to deal with this is to add explicit tense variables to $\mathcal{W}$, so that ${ }^{\top} \mathcal{W}_{t} \phi^{\top}$ is true at $t^{\prime}$ iff $\phi$ is true in the closest world at time $t$; and, to get the future shifting effect, we could add a presupposition that $t$ must be in the future of whatever time ${ }^{\ulcorner} \mathcal{W}_{t} \phi^{\urcorner}$is evaluated at. But see Cariani (2019) also for another approach. 


$$
\llbracket P A S T \phi \rrbracket^{w, t}=1 \text { iff } \exists t^{\prime}<t: \llbracket \phi \rrbracket^{w, t^{\prime}}=1
$$

I assume the standard three-valued semantics for negation:
a. $\quad \llbracket \neg \phi \rrbracket^{w, t, f, \mathcal{I}}=1$ iff $\llbracket \phi \rrbracket^{w, t, f, \mathcal{I}}=0$
b. $\quad \llbracket \neg \phi \rrbracket^{w, t, f, \mathcal{I}}=0$ iff $\llbracket \phi \rrbracket^{w, t, f, \mathcal{I}}=1$.
c. $\llbracket \neg \phi \rrbracket^{w, t, f, \mathcal{I}}=\#$ iff $\llbracket \phi \rrbracket^{w, t, f, \mathcal{I}}=\#$.

I assume the standard Kaplanian definition of truth at a context:

Truth at a context. $\llbracket \phi \rrbracket^{c}=\llbracket \phi \rrbracket^{w_{c}, t_{c}, f_{c}, \mathcal{I}_{c}}$

And finally I assume entailment is preservation of truth at a context:

Diagonal validity. $\phi_{1}, \ldots, \phi_{n} \models \overline{\bar{c}} \psi$ iff whenever $\llbracket \phi_{1} \rrbracket^{c}=\ldots=\llbracket \phi_{n} \rrbracket^{c}=1$ then $\llbracket \psi \rrbracket^{c}=$ 1.

\subsection{Delivering Openness, Past Settledness and Asymmetry}

This package delivers our three features:

Openness. $\diamond \phi, \diamond \neg \phi \leadsto \nabla \mathcal{W} \phi$

Past Settledness. PAST $\phi \leadsto$ PAST $\mathcal{W} \phi$

Scopelessness. $\neg \mathcal{W} \phi \leftrightarrow \mathcal{W} \neg \phi$

Let's now see why at an informal level. (See Appendix B for the proofs.)

Openness holds because if it is unsettled whether $\phi$ will happen, then the actual unsettled world, the closest world to itself, does not settle whether $\phi$ happens. Return to Monday in our sea battle case. At this point it is possible that there will be a sea battle on Tuesday and possible that there won't. So the actual un-

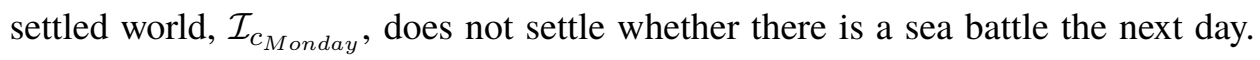
Of course, $\mathcal{I}_{c_{\text {Monday }}}$ is the closest unsettled world to itself. So the closest unsettled world doesn't settle whether there will be a sea battle on Tuesday. This means (24) below is indeterminate:

$\mathcal{W}$ (There is a sea battle on Tuesday) 
Past Settledness holds because, once $\phi$ becomes settled, the actual unsettled world, again the closest world to itself, settles $\phi$. Move forward to Tuesday in the sea battle case. Now the sentence

There was a sea battle on Tuesday.

is true in the context of utterance. That means the unsettled world of the context we now occupy, $\mathcal{I}_{C_{\text {Tuesday }}}$, settles that there was a sea battle on Monday. Now consider whether the following is true:

$$
P A S T \mathcal{W} \text { (There is a sea battle on Tuesday). }
$$

Take some arbitrary time $t^{\prime}$ in the past : what is the closest world to $\mathcal{I}_{c_{\text {Tuesday }}}$ at $t^{\prime}$ ? More precisely, what is $s\left(\mathcal{I}_{c_{\text {Tuesday }}}, f_{c}\left(w_{c}, t^{\prime}\right)\right)$ ? Since $f_{c}\left(w_{c}, t^{\prime}\right)$ is just the set of all worlds, by Overlap, it must be $\mathcal{I}_{c}$ itself. But, since (36) is true, we know that $\mathcal{I}_{c_{\text {Tuesday }}}$ settles that there was a sea battle. So there's some past time $t^{\prime}$ when the closest world to $\mathcal{I}_{C_{\text {Tuesday }}}$ settled that there is a sea battle on Tuesday. This suffices to make (25) true.

Finally, Scopelessness is valid because, if the actual unsettled world is determinately not one where $\phi$ holds, then it is one where $\neg \phi$ is determinately true.

Suppose the following is true:

$$
\neg \mathcal{W} \text { (I eat another cookie). }
$$

This means that the following sentence is false: $\mathcal{W}($ I eat another cookie)

(38) is false iff the closest world to the unsettled world of the context is one where I do not eat another cookie. In other worlds, $\mathcal{I}_{c}$ settles that I do not eat another cookie. But this is exactly what it takes for the sentence: $\mathcal{W}_{\neg(\text { I eat another cookie })}$

to be true. So if ${ }^{\ulcorner} \neg \mathcal{W} \phi \phi^{\top}$ is true, then so is ${ }^{\ulcorner} \mathcal{W} \neg \phi{ }^{\top}$. 


\section{A New Conditional Analysis}

Now that we have introduced $\mathcal{W}$, I can give my semantics for abilities. On my theory, ' $\mathrm{S}$ can $\phi$ ' is true iff, for some available action $\alpha$, if $\mathrm{S}$ tries to $\alpha$ then $\mathcal{W}(\mathrm{S}$ $\phi$ 's). ${ }^{34}$ In this section I lay out the view on an intuitive level; in the next, I provide all the formal details.

Stating my semantics in terms of $\mathcal{W}$ allows us to capture the control intuition. Consider:

I can hit the bullseye on this throw.

Assume the actions available to me are a set of tryings, a set of things I try to do. For (6) to be true, there must be some action available to me that settles that I hit the bullseye, in the closest unsettled world where I perform it. But it is hard for an unsettled world to settle that I hit the bullseye: all the worlds in it must agree that I hit the bullseye. This is the sense in which hitting the bullseye must be in my control. $^{35}$

But two other components must be explained, before we fully understand the analysis. When is an action is available, in my sense? We don't need a full account to settle questions about the logic of 'can', but here are some general constraints. I think of an agent's available actions as their options. ${ }^{36}$ And, for simplicity at least, we can typically think of options as a set of tryings: ${ }^{37}$ for example, in Unreliable Dartboard my available options might be trying to hit the top and trying to hit the bottom. This allows us to move the notion of trying out of the semantics and into the pragmatics: going forward I simply say that ${ }^{\ulcorner} \mathrm{S}$ can $\left.\phi\right\urcorner$ is true iff, for some available action $\alpha$, if $\mathrm{S}$ does $\alpha$, then $\mathcal{W}(\mathrm{S} \phi$ 's); the context of utterance determines whether

\footnotetext{
${ }^{34}$ As mentioned, this semantics builds on the account of Mandelkern et al. (2017). That view in turn builds on the conditional semantics of Lehrer (1976), Cross (1986) and Thomason (2005).

${ }^{35}$ My explanation of the control intuition is close in spirit to the stit analysis of Horty and Belnap (1995) and Horty (2001); however, as we will see, it interacts very differently with negation and tense.

${ }^{36}$ One might worry that this is circular: if $\phi$ is an option for you doesn't that entail you are able to $\phi$ ? This would indeed be a worry if we were trying to provide a reductive analysis of abilities. But this is not our project: we are rather trying to say enough about ability attributions to predict what inference patterns involving abilities are valid. (Although the approach sketched in footnote 27 may provide the starting point for a reductive analysis.) For precedents of this kind of attitude, see Stalnaker (1968) on conditionals and Williamson (2000) on the logic of knowledge.

${ }^{37}$ Hedden $(2012,2015)$, for instance, endorses a view like this for independent reasons.
} 
the available actions are tryings and what tryings they may be. ${ }^{38}$

Second, though we know when ' $\mathrm{S}$ can $\phi$ ' is true, we do not yet know when it is false. When it is not the case that some action settles that $\mathrm{S} \phi$ 's, the theory says that 'S can $\phi$ ' is not true, that is, either false or indeterminate. But there are two ways for this to happen: it could be that every action settles that $\mathrm{S}$ does not $\phi$; or it could be that some actions do not settle whether or not S $\phi$ 's. And we do not yet know what particular value the ability claim gets in each situation. ${ }^{\ulcorner} \mathrm{S}$ can $\phi{ }^{\top}$ can fail to be true in two ways: it could be false or indeterminate.

To better appreciate this point, take again:

I can hit the bullseye on this throw.

Consider this first as said by Alice, an expert dartplayer who has mastered an unspectacular shot that always yields a bullseye and is learning some trick shots which are less reliable. For her some action settles that it is determinately true that Alice will hit the bullseye; so (6) is true as said by Alice. But now consider two other, less expert players. Billy knows how to perform some shots which will definitely lead him not to hit the bullseye; but none of his remaining shots settle whether he hits the bullseye. And Carol is so hopeless that she cannot even hit the board: every throw open to her settles that her dart misses the board entirely. While it cannot be true, the view so far does not tell us whether (6) is false or indeterminate, when said by Billy or Carol.

\footnotetext{
${ }^{38}$ Moving tryings out of the semantics also helps solve two problems for the traditional formulation of the conditional analysis. Firstly, on the standard formulation, the ability to try is predicted to be trivial. If someone tries to $\phi$, then, trivially, they try to $\phi$. But trying is not trivial: comatose patients, for instance, arguably cannot try to get up, open their eyes or indeed do anything. Secondly, trying creates problems when we ascribe abilities to non-agential objects. We can say, truly, that a flower can follow the sun, that an elevator can carry 300 pounds or that a rock can tip the balance. In none of these cases are we considering what would happen if such objects tried to do something.

My version of the conditional analysis, which does not mention the notion of trying in the semantics, has the resources to avoid these objections. We can generalise the account by saying 'can' quantifies over answers to some salient question. When the question is What option will S choose?, then 'can' quantifies over the available actions. But in other cases, we will get new and improved predictions. For the comatose patient, the question might be What will happen to the patient?; and presumably any answer to that question (consistent with the background facts) will entail, for instance, that the patient does not try to open their eyes. For inanimate objects, the question could concern what happens to the object: questions like Will the rock be placed on the scale? or Will the flower be planted outside? yield plausible predictions for those cases.
} 
I give a Strong Kleene theory of how indeterminacy projects here: whenever no action settles that $\mathrm{S}$ does $\phi$ and some action makes it indeterminate whether $\mathrm{S} \phi$ 's, then ${ }^{r} \mathrm{~S}$ can $\phi$ ' is indeterminate. Return to (6) as said by Alice. Intuitively, it does not matter that some other actions leave it unsettled that she will hit the bullseye: there is a surefire way for her to hit the bullseye, so the existential claim should be true. (6) should come out false as said by Carol: all actions determinately lead her to not hit the bullseye. And, as said by Billy, I say (6) is indeterminate: no action determines he will hit the bullseye; but some actions fail to determine whether he will either way. As I spell out in the next section, this is exactly what the Strong Kleene approach predicts: ${ }^{\ulcorner} \mathrm{S}$ can $\left.\phi\right\urcorner$ is indeterminate just in case no action settles that $\mathrm{S} \phi$ 's and some action leaves it unsettled whether $\mathrm{S} \phi$ 's.

So that is the view: ' $\mathrm{S}$ can $\phi$ ' says that, for some available action, the closest world where that action is performed settles that $S \phi$ 's; available actions are our options, construed as a set of tryings; and it is determinate when either some action settles that $S \phi$ 's or every action settles that $S \neg \phi$ 's.

\section{The Formal Details}

I work up to the full semantic entry for ${ }^{\top} \mathrm{S}$ can $\phi$ ' in two steps. First, I focus on the truth-conditions for 'can', formalising the notion of an available action and clarifying the conditional element of 'can'. Then I spell out more precisely the Strong Kleene determinacy conditions.

\subsection{The Semantics}

To simplify, propositions about actions stand in for actions. I represent the available actions using a function $\mathcal{A}$ that takes a world and a time and yields a set of propositions. $\mathcal{A}(w, t)$ is the set of actions available to the subject at $w$ and $t$.

I assume that the set of available actions represents a set of mutually exclusive and exhaustive options for the agent: in every circumstantially accessible world, the agent performs exactly one of the available actions. Thinking of actions as propositions allows us to formulate this idea in a natural way. The set of available actions is a partition of the circumstantially possible worlds: every circumstantially accessi- 
ble world is a member of exactly one proposition in $\mathcal{A}(w, t)$ (and $\mathcal{A}(w, t)$ does not contain the empty set). ${ }^{39}$ Call this assumption Partitionality. ${ }^{40}$ Importantly, since the actual world is always circumstantially accessible to itself, the actual world is always a member of at least one available action.

To fully spell out my conditional analysis, we need to be clear on how the conditional works. I give it a restrictor semantics, a la Kratzer (1981, 2012): a conditional restricts the domain for a modal in the consequent to worlds where the antecedent is true. ${ }^{41}$ More precisely, where $f^{\mathbf{A}}$ is the function such that $f^{\mathbf{A}}(w)=f(w) \cap \mathbf{A}$, the conditional has the following truth-conditions.

$$
\llbracket \text { if } \phi, \psi \rrbracket^{w, t, f, \mathcal{I}}=1 \text { iff } \llbracket \psi \rrbracket^{w, t, f \llbracket \phi \rrbracket^{t, f, \mathcal{I}}, \mathcal{I}}=1
$$

When $\mathcal{W}$ is embedded in the consequent, a restrictor conditional restricts the modal base argument to the selection function. We said that ${ }^{\circ} \mathcal{W} \phi{ }^{\prime}$ is true iff the closest (possibly indeterminate) world to the actual unsettled world is one where $\phi$ is true. 'if $\phi, \mathcal{W} \psi\urcorner$ is true iff the closest world to the actual unsettled world where $\phi$ is true is one where $\psi$ is true. More precisely:

$$
\begin{aligned}
& \llbracket \text { if } \phi, \mathcal{W} \psi \rrbracket^{w, t, f, \mathcal{I}}=1 \text { iff } \llbracket \mathcal{W} \psi \rrbracket^{w, t, f^{\llbracket \phi \rrbracket^{t, f, \mathcal{I}}}, \mathcal{I}=1} \\
& \text { iff } s\left(\mathcal{I}, f \llbracket \phi \rrbracket^{t, f, \mathcal{I}}(w, t)\right) \subseteq \llbracket \psi \rrbracket^{t, f^{\llbracket \phi \rrbracket^{t, f, \mathcal{I}}}, \mathcal{I}}=1
\end{aligned}
$$

We can now state precisely my truth-conditions from $\S 4$. The rough idea was that ${ }^{\top} \mathrm{S}$ can $\left.\phi\right\urcorner$ is true iff for some action, in the closest world where $\mathrm{S}$ does that action, $\mathrm{S} \phi$ 's. This is spelled out as follows:

$$
\begin{aligned}
& \llbracket \mathrm{S} \text { can } \phi \rrbracket^{w, t, f, \mathcal{I}}=1 \text { iff for some } \alpha \in \mathcal{A}(w, t): \llbracket \mathcal{W}(\mathrm{S} \phi ' s) \rrbracket^{w, t, f^{\alpha}, \mathcal{I}}=1 \\
& \text { i.e. iff for some } \alpha \in \mathcal{A}(w, t): s\left(\mathcal{I}, f^{\alpha}(w, t)\right) \subseteq \llbracket \mathrm{S} \phi ' s \rrbracket^{t, f^{\alpha}, \mathcal{I}}=1
\end{aligned}
$$

\footnotetext{
${ }^{39}$ This is a standard move in the literature in deontic modals. See for instance Cariani (2013).

${ }^{40}$ This condition can actually be weakened to the following:

Sub-partitionality. There is some subset $\mathcal{A}^{\prime}$ of $\mathcal{A}(w, t)$ s.t. $\mathcal{A}^{\prime}$ partitions the circumstantially possible worlds at $w, t$ (and $\mathcal{A}(w, t)$ does not contain the empty set).

This weaker assumption suffices to capture the validities and invalidities I want; but it has the added advantage of invalidating the inference from ${ }^{\ulcorner} \mathcal{W} \phi{ }^{\urcorner}$to ${ }^{\ulcorner}$cannot $\neg \phi{ }^{\urcorner}$. For simplicity, I will continue to take Partionality as my official assumption. Thanks to Annina Loets for discussion here.

${ }^{41}$ Kratzer assumes bare conditionals involve a tacit epistemic 'must' in the consequent.
} 


\subsection{Determinacy Conditions}

On a Strong Kleene approach to disjunction, ${ }^{r} \phi$ or $\left.\psi\right\urcorner$ is determinate when we have enough information to determine a unique truth-value using the classical truth-table for 'or'. If at least one of $\phi$ and $\psi$ is true, ${ }^{\ulcorner} \phi$ or $\left.\psi\right\urcorner$ is true; if both are false, ${ }^{\ulcorner} \phi$ or $\psi$ ' is false; in all remaining cases it is indeterminate. This idea carries over to existential quantifiers: an existentially quantified sentence is true if it has a true instance; it is false if it has only false instances; and indeterminate otherwise.

Now apply this to 'can'. I say that ' $\mathrm{S}$ can $\phi$ ' is true when, for some available $\alpha$, 'If $\mathrm{S} \alpha$ 's, $\mathcal{W}(\mathrm{S} \phi \text { 's })^{7}$ is true; ' $\mathrm{S}$ can $\phi$ ' is false when, for every available $\alpha$, ' If $\mathrm{S}$ $\alpha$ 's, $\mathcal{W}\left(\mathrm{S} \phi{ }^{\prime} \mathrm{s}\right)^{\urcorner}$is false; and is indeterminate, otherwise. Spelled out precisely:

$\llbracket \mathrm{S}$ can $\phi \rrbracket^{w, t, f, \mathcal{I}}$ is determinate only if either

a. $\quad$ there is some $\alpha \in \mathcal{A}(w, t)$ such that $\llbracket \mathcal{W}(\mathrm{S} \phi$ 's $) \rrbracket^{w, t, f^{\alpha}, \mathcal{I}}=1$;

b. $\quad$ or for all $\alpha \in \mathcal{A}(w, t), \llbracket \mathcal{W}(\mathrm{S} \phi$ 's $) \rrbracket^{w, t, f^{\alpha}, \mathcal{I}}=0$.

Combine this with the truth-conditions and we have full statement of the view:

a. $\llbracket \mathrm{S}$ can $\phi \rrbracket^{w, t, f, \mathcal{I}}$ is determinate only if either

(i) there is some $\alpha \in \mathcal{A}(w, t)$ such that $\llbracket \mathcal{W}(\mathrm{S} \phi$ 's $) \rrbracket^{w, t, f^{\alpha}, \mathcal{I}}=1$;

(ii) or for all $\alpha \in \mathcal{A}(w, t)$, $\mathcal{W}(\mathrm{S} \phi$ 's $) \rrbracket^{w, t, f^{\alpha}, \mathcal{I}}=0$.

b. If determinate, $\llbracket \mathrm{S}$ can $\phi \rrbracket^{w, t, f, \mathcal{I}}=1$ iff for some $\alpha \in \mathcal{A}(w, t): \llbracket \mathcal{W}(\mathrm{S}$ $\phi ' s) \rrbracket^{w, t, f^{\alpha}, \mathcal{I}}=1$

i.e. iff for some $\alpha \in \mathcal{A}(w, t): s(\mathcal{I}, f(w, t) \cap \alpha) \subseteq \llbracket \mathrm{S} \phi$ 's $\rrbracket^{t, f^{\alpha}, \mathcal{I}}$

\section{Predictions}

I distilled the tension involving Success into three data points about ability modals:

Validity of Past Success. PAST (S $\phi) \leadsto P A S T$ (S can $\phi)$

Validity of Can't-entails-won't. $\neg(\mathrm{S}$ can $\phi) \leadsto \neg(\mathrm{S} \phi)$

Invalidity of $\vee$-Success. $\mathrm{S} \phi \vee \mathrm{S} \psi \rightsquigarrow \mathrm{S} \operatorname{can} \phi \vee \mathrm{S} \operatorname{can} \psi$

My semantics delivers each of these predictions: it explains why Success is so close 
to being valid, despite having counterexamples.

\subsection{Failure of $\vee$-Success}

$\vee$ - Success fails on my theory because $\mathrm{S}$ may end up $\phi$-ing even though no available action settles whether $\mathrm{S} \phi$ 's.

Return to Unreliable Dartboard. What are the available actions? A natural choice is I try to hit the top and I try to hit the bottom. And since I am a bad dartplayer, neither settles what happens. I may get lucky: in some worlds I succeed in doing what I try to do. But I also may not: in some worlds I do exactly the opposite of what I try.

Let's spell this out a little more precisely. Assume there are just four worlds, one for each of these situations; call them $w_{\text {try } T \wedge \text { hit } B}, w_{\text {try } B} \wedge$ hit $T, w_{\text {try } T \wedge h \text { hit } T}$ and $w_{\text {try } B} \wedge$ hit $B$. These are all and only the scenarios compatible my current situation and so the unsettled world of the context $\mathcal{I}_{c}$ contains just these four worlds:

$$
\mathcal{I}_{c}=\left\{w_{\text {try } T \wedge \text { hit } B}, w_{\text {try } B \wedge \text { hit } T}, w_{\text {try } T \wedge \text { hit } T}, w_{\text {try } B} \wedge \wedge \text { hit } B\right\}
$$

The closest unsettled world where I try to hit the top is the set $\left\{w_{\text {try } T \wedge \text { hit } B}, w_{\text {try }} T \wedge\right.$ hit $\left.T\right\}$. Crucially, this world does not settle whether I hit the top or the bottom: it leaves both open. The closest world where I try to hit the bottom is $\left\{w_{\text {try } B} \wedge\right.$ hit $\left.T, w_{\text {try } B \wedge \text { hit } B}\right\}$ and this also leaves the outcome unsettled.

So, given our semantics for $\mathcal{W}$ and for the conditional, for both available actions $\alpha$, it is indeterminate whether I will hit the top, if I perform $\alpha$ and indeterminate whether I will hit the bottom, if I perform $\alpha$. Given Strong Kleene projection, both of the following must be indeterminate:

I can hit the top of the dartboard on this throw.

I can hit the bottom of the dartboard on this throw.

In general, when both disjuncts of a disjunction are indeterminate, then so is the whole disjunction. So the conclusion of our inference,

(48) I can hit the top of the dartboard on this throw or I can hit the bottom of the dartboard on this throw. 
is indeterminate and Unreliable Dartboard is a counterexample to Success: I am going to either hit the top or the bottom of the dartboard; but it is indeterminate whether I can hit the top and whether I can hit the bottom.

In fact, as I show in the Appendix, examples like these are the only kinds of cases where Success fails: if the premise of Success is true, the conclusion is at worst indeterminate - never false. In other words, in the terminology of von Fintel (1999), Success is Strawson-valid: whenever the premise of Success is true and the conclusion is determinate, then the conclusion is true.

\subsection{Past Success}

I will give the flavour of why Past Success holds by showing how the inference goes through in Unreliable Dartboard.

Imagine that as a matter of fact the following is now true in our context:

I hit the top of the dartboard a few moments ago.

This means that it is settled whether I hit the dartboard: the unsettled world of the context, $\mathcal{I}_{c}$, contains only worlds where I hit the dartboard a few moments ago.

Trying to hit the top and trying to hit the bottom were the actions available to me, so I must have done one of the two. Suppose as a matter of fact I tried to hit the top. So the unsettled world of the context also settles that I tried to hit the top a few moments ago.

Now, consider:

(50) I was able to hit the top of the dartboard a few moments ago.

To see if this is true, we need to ask, was there some $\alpha$ available to me such that in the closest world where I performed it, it is settled that I hit the top?

The answer to this question is yes. Consider the actual unsettled world of our present context. There just a few moments ago, at $t^{\prime}$ say, I performed an available action - I tried to hit the top. So $\mathcal{I}_{c}$, the actual unsettled world, is $s\left(\mathcal{I}_{c}, f_{c}\left(w_{c}, t^{\prime}\right)\right)$, the closest unsettled world to $\mathcal{I}_{c}$ at $t^{\prime}$ where I tried to hit the top. And we already know that there it is settled that I did in fact hit the top. So trying to hit the top 
witnesses the truth of (50): the actual unsettled world is the closest world where I perform it; and we know that I actually did hit the top.

\subsection{Can't-entails-won't}

Can't-entails-won't is valid because ' $\mathrm{S}$ cannot $\phi$ ' says that for all available actions $\alpha$, the closest world where $\mathrm{S} \alpha$ 's settles that $\mathrm{S}$ does not $\phi$. Since some available action must always be performed, this ensures that $\mathrm{S} \phi$ 's.

Let's start by seeing why ' $\mathrm{S}$ cannot $\phi$ ' means what it does on my view. Recall the three-valued account of negation: ${ }^{r} \neg \phi{ }^{\top}$ is true when $\phi$ is false; is false when $\phi$ is true; and is indeterminate when $\phi$ is too. So ${ }^{\ulcorner} \mathrm{S}$ cannot $\phi^{\top}$ is true iff ${ }^{\mathrm{r}} \mathrm{S}$ can

$\phi\urcorner$ is false; is false when ' $\mathrm{S}$ can $\phi^{\top}$ is true; and is otherwise indeterminate. Given my theory, this means that ${ }^{\ulcorner} \mathrm{S}$ cannot $\phi$ ' is true when every available action settles that $\mathrm{S}$ does not $\phi$; is false when some available action settles that $\mathrm{S}$ does $\phi$; and is otherwise indeterminate.

To see this better, consider: I cannot hit the bullseye.

The precise truth-value of this claim depends on the truth-value of the claim: I can hit the bullseye.

On my theory, (52) is true iff, for some available action $\alpha$, the closest world where I perform $\alpha$ settles that I hit the bullseye. (52) is false iff, for every available $\alpha$, the closest world where I perform $\alpha$ is one where I do not hit the bullseye. In the remaining cases, (52) is indeterminate. This means that (51) is true iff, for every available $\alpha$, the closest world where I perform $\alpha$ is one where where I do not hit the bullseye.

It is now easy to see why Can't-entails-won't holds. If (51) is true, then, for all available actions, the closest unsettled world where I do that action is one where I do not hit the bullseye. So every action settles that I do not hit the bullseye. Now remember that the available actions are my options: no matter how things go, I will perform exactly one of the available actions. So if (51) is true, then, no matter how things go, no matter what I action I perform, I end up not hitting the bullseye. But 
then the actual indeterminate world must already settle that I will not the bullseye and so it must be true that I will not hit the bullseye. More generally, when 'S cannot $\phi^{\urcorner}$is true, every available action ensures that $S$ does not $\phi$. And since $S$ always performs an available action, it follows that $\mathrm{S}$ does not $\phi$.

\section{The Broader Picture}

We have shown how to reconcile our three data points. Before concluding, I want to reflect on why these data are so central to an account of ability; and then examine how the account treats a battery of other standard data involving ability modals.

In the Introduction, I noted that ability modals are governed by the control intuition: saying, for instance, that you can hit a home run communicates that doing so is in your control. This is one of the central features of ability modals. It distinguishes them from their closest cousins, the pure circumstantial or metaphysical modals, which do not require any notion of control. For example in Unreliable Dartboard, I can say:

I could hit the dartboard.

(54) I might hit the dartboard.

These circumstantial modal readings are unquestionably true, even though hitting the dartboard is not in my control. For particularly extreme cases, we might look to lotteries. Even when the odds are stacked against me, I am surely entitled to say:

$$
\text { I could win! }
$$

But the claims:

I can win.

(57) I am able to win.

strike me as bizarre, on their most natural reading where the modal picks out ability.

So control is central to ability. My puzzle is about how to make sense of control; my data show that it is much harder than one might have thought. Whatever 
control amounts to, one might have thought that at a minimum success does not establish control. I have argued this is ultimately correct, but hard to capture. Any analysis of control must also validate Past Success and Can't-entails-won't; given their interconnections, this is hard to do while also invalidating Success. So I take solving my puzzle to be a very important empirical advantage of the account: my notion of control that is faithful to the motivating intuitions and makes sense of Past Success and Can't-entails-won't.

But my empirical coverage is not limited to the central puzzle. ${ }^{42}$ Firstly, accounting for this puzzle allows me to capture the other distinctive logical features of ability modals. Mandelkern et al. (2017) stress that a plausible account of ability modals must predict a plausible meaning for the dual, cannot not. Consider:

I cannot not have another cigarette.

Mandelkern et al. (2017) observe that cannot not is strong because it expresses compulsion - (58) says that I will have a cigarette no matter what. This is surprising, given that 'can' is also in a certain sense strong. Many accounts designed to capture this strength, like the boxy view, wrongly predict the dual is weak. ${ }^{43}$

My account gives the dual a plausible meaning, in line with that of Mandelkern et al. (2017). Given my entries for 'can' and 'not', we can derive the following entry for 'cannot not':

a. $\llbracket$ cannot not $\phi \rrbracket^{w, t, f, \mathcal{I}}$ is determinate only if either

(i) there is some $\alpha \in \mathcal{A}(w, t)$ such that $\llbracket \mathcal{W} \neg \phi \rrbracket^{w, t, f^{\alpha}, \mathcal{I}}=1$;

(ii) or for all $\alpha \in \mathcal{A}(w, t), \llbracket \mathcal{W} \neg \phi \rrbracket^{w, t, f^{\alpha}, \mathcal{I}, \mathcal{A}}=0$.

b. If determinate, $\llbracket$ cannot $\phi \rrbracket^{w, t, f, \mathcal{I}}=1$ iff for all $\alpha \in \mathcal{A}(w, t): \llbracket \mathcal{W} \neg \phi \rrbracket^{t, f^{\alpha}, \mathcal{I}} \neq$ 1

i.e. iff for all $\alpha \in \mathcal{A}(w, t): s(\mathcal{I}, f(w, t) \cap \alpha) \nsubseteq \llbracket \neg \phi \rrbracket^{t, f^{\alpha}, \mathcal{I}}$

i.e., given the determinacy conditions, iff for all $\alpha \in \mathcal{A}(w, t): s(\mathcal{I}, f(w, t) \cap$

$\alpha) \subseteq \llbracket \phi \rrbracket^{t, f^{\alpha}, \mathcal{I}}$

Focus on the truth-conditions here: ${ }^{\top} \mathrm{S}$ cannot $\phi{ }^{\prime}$ is true iff $\mathrm{S}$ will $\phi$, no matter

\footnotetext{
${ }^{42}$ Thanks to an anonymous reviewer for pressing me to consider a wider range of data.

${ }^{43}$ See Mandelkern et al. (2017) for these arguments.
} 
what action they take. This is an appropriate meaning for a sentence like (58). But furthermore, since I deliver Can't-entails-won't, my view has the advantage that it predicts the dual is factive: 'cannot not $\phi$ ' entails $\phi .{ }^{44}$

Unsurprisingly, I also account for Kenny's observation that ability does not distribute across disjunction. That is, the following principle fails:

$$
\mathrm{S} \operatorname{can}(\phi \vee \psi) \not \models \mathrm{S} \text { can } \phi \vee \mathrm{S} \operatorname{can} \psi
$$

In Unreliable Dartboard, if I try to hit the dartboard, I will; so some action available to me, namely trying to hit the dartboard, ensures I either hit the top or bottom of the dartboard, giving us: ${ }^{45}$

I can hit the dartboard.

But as we have already seen, I predict that it is not true that: ${ }^{46}$

(10) I can hit the top of the dartboard or I can hit the bottom of the dartboard.

I also account for an important non-logical feature of ability claims, their pervasive context-sensitivity. My account ties ability to circumstantial possibility. The available actions, whatever they are, must partition the circumstantial possibilities.

\footnotetext{
${ }^{44}$ For the reasons discussed in footnote 20, Mandelkern et al. (2017) delivers this prediction only if it validates Success.

${ }^{45}$ To control for free choice effects, I do not formulate (61) with an overt disjunction.

${ }^{46}$ On the approach of Mandelkern et al. (2017), specific ability does distribute across disjunction. However, they claim that, since their account supervaluates over admissible selection functions, neither of the disjuncts of (10) is determinately true and that they thereby capture something like Kenny's intuition. This is not accurate: for one of the disjuncts to fail it must also be that the agent does not try to perform any available action.

To see why, first note that any admissible selection function for their conditional obeys Strong Centering: the closest A-world to $w$ is $w$ itself, if $\mathbf{A}$ holds at $w$. Now in cases like Unreliable Dartboard, I will either hit the top or the bottom. Suppose I do try to do some available action $\alpha$. If I hit the top, then by Strong Centering, the closest world where I try to perform $\alpha$ on every admissible selection function is the actual world where I hit the top; if I hit the bottom, by the same reasoning, the closest world where I perform $\alpha$ on every admissible selection function is the actual world where I hit the top. In either case, either ${ }^{\top} \mathrm{I}$ can hit the top ${ }^{\urcorner}$is true on every admissible selection function or 'I can hit the bottom' is.

It is not particularly plausible in this situation that I do not try to perform an available action here. But moreover, as we saw in footnote 20 , maintaining that no available action is attempted means that Past Success and Can't-entails-won't fail. So I think my account, which says both disjuncts and the disjunction are indeterminate, better captures Kenny's data.
} 
And what you are able to do, on my account, is in part determined by the available actions. Since the circumstantial possibilities are a matter of the context, so too is what one's abilities are.

Take the example of Alice, an expert in French cooking. We might say of her:

Alice can make ratatouille.

By this we mean she has the requisite know how and skill. But suppose we lack the ingredients for ratatouille. Now we can also say:

(63) Alice can't make ratatouille; she doesn't have the ingredients.

This context-sensitivity seems to track the context-sensitivity of circumstantial possibility. If we are not attending to what ingredients are on hand and you ask what dishes Alice knows how to make, I could say:

(64) Alice could make ratatouille (or bouillabaisse or beef bourguignon...).

But ask instead what we should make for dinner tonight and (64) sounds false.

My account predicts this. When something is circumstantially impossible, it is inconsistent with the available actions. So when we hear the true reading of (62), we must be in a context where (64) is true and ratatouille-making is a circumstantial possibility. When instead we are in the context where (64) is false, then (62) is false and instead (63) is true. The contextual variation in what counts as circumstantially possible produces variation in what we count as being able to do.

This kind of context-sensitivity also helps deal with some apparent counterexamples to my analysis of control. ${ }^{47}$ One might think that my analysis of control makes it too strong. I might tell my partner:

I can pick you up from work.

But of course, it is possible my car might break down, in which case I would not. This does not seem to undermine my claim in (65), especially if I go on to successfully drive my partner home. But my analysis might seem to say it does — if it's

\footnotetext{
${ }^{47}$ Thanks to Ginger Schultheis for discussion here.
} 
possible the car might break down, then no action will determinately lead to me picking up my partner.

Of course the solution is that, in normal contexts, we hold fixed that the car will not break down. Notice that I could equally comfortably have said:

I will pick you up from work.

This would certainly be in tension with the possibility of a breakdown, if that was indeed a possibility. ${ }^{48}$ I cannot say things like:

(67) The car could break down, but I will pick you up from work.

The natural conclusion here is that we hold fixed the fact that the car will not break down and so it is not circumstantially possible in most cases. Once that is granted, (65) poses no problem to my account.

Finally, this tie to circumstantial possibility equips me to account for some more traditional counterexamples to conditional analyses of ability. Consider the case of Larry, from Lehrer (1968), who has a pathological fear of blood-red candy. Were he offered a bowl, he could not be induced to take any. We might readily say of him:

He is not able to take a candy.

As Lehrer notes, Larry could easily take a candy if he tried; but it is his inability to even try that accounts for his inability to take a candy.

Nonetheless, I correctly predict (68) is false. It is also circumstantially impossible for Larry to take a candy, on at least one resolution of circumstantial possibility. Consider:

Larry could take a candy.

The most prominent reading is straightforwardly false - the circumstances determine that he won't take one. Since on my view the available actions partition the circumstantial possibilities, all will entail he does not take a candy. ${ }^{49}$

\footnotetext{
${ }^{48}$ I say in tension, as the exact nature of the tension depends exactly on one's views about 'will'.

${ }^{49}$ Note that I get the further desirable prediction that he cannot even try.
} 
Circumstantial possibility is not the only source of context-sensitivity on my account. How to partition circumstantial worlds into actions is itself a contextsensitive matter, as Cariani (2013) and Mandelkern et al. (2017) among others have argued. Nonetheless, it is noteworthy that many existing examples of the contextsensitivity of ability can, on my view, be traced to an existing form of contextsensitivity that is relatively well understood.

\section{Conclusion}

We started with a dilemma. There is a compelling case for Success: Past Success is valid and so is Can't-entails-won't; and both are extremely close to Success. But there are also compelling counterexamples to $\vee$-Success and hence to Success itself. How can we enjoy the virtues of Success without being stuck with its vices too?

I stated my theory in terms of $\mathcal{W}$-conditionals — on my semantics ' $\mathrm{S}$ can $\phi$ ' says that, for some available action $\alpha$, if $\mathrm{S} \alpha$ 's, $\mathcal{W}(\mathrm{S} \phi$ 's) - and showed how it reconciled all three properties. Following a venerable tradition, $\mathcal{W}$ requires settledness: ${ }^{\ulcorner} \mathcal{W} \phi{ }^{\top}$ is true only when $\phi$ is settled; and is false only when $\left.{ }^{\ulcorner} \neg \phi\right\urcorner$ is settled. But past $\mathcal{W}$ claims require mere past truth, not past settledness. My view of abilities inherits similar properties. ' $\mathrm{S}$ can $\phi$ ' requires that some action settles that $\mathrm{S} \phi$ 's. Even if $S$ goes on to $\phi$, this does not mean it is now settled; and so my account invalidates $\vee$-Success. But, once $\mathrm{S}$ does $\phi$, past truth suffices to establish past ability, and so I

validate Past Success. Finally, 'S cannot $\phi$ ' requires every available action to settle that $\mathrm{S}$ does not $\phi$ and so entails $\mathrm{S}$ does not $\phi$, giving us Can't-entails-won't. 


\section{A Proofs of Claims in \$1.3}

We first prove the following claim about the Boxy analysis:

Fact 1. Success is valid on the Boxy analysis just in case $\{w\} \in \mathcal{A}(w)$.

Proof. $\Rightarrow$ : Suppose for some $w\{w\} \notin \mathcal{A}(w)$. We construct a countermodel by letting $\llbracket p \rrbracket=\{w\}$. Now we have $\llbracket p \rrbracket^{w}=1$, by our choice of evaluation function; but $\llbracket$ Can $p \rrbracket^{w}=0$, since there is no proposition in $\mathcal{A}(w)$ that entails $p$.

$\Leftarrow$ : Suppose $w \in \mathcal{A}(w)$. Now suppose $\llbracket \phi \rrbracket^{w}=1$. We can see some element of $\mathcal{A}(w)$ entails $\phi$, namely $\{w\}$. So $\llbracket$ Can $\phi \rrbracket^{w}=1$.

Moreover, the reader can easily adapt the above proof to show the same condition characterises Can't-entails-won't.

Next we prove the following claim about the analysis of Mandelkern et al. (2016):

Fact 2. Success is valid on the Mandelkern et al. analysis just in case $w \in S$ tries to $\alpha$, for some $\alpha \in \mathcal{A}(w)$.

Before proving this, it is important to recall that Stalnakerian selection functions obey the following conditions:

Minimality. $f(\mathbf{A}, w) \in \mathbf{A}$, when $\mathbf{A} \neq \varnothing$.

Strong Centering. $f(\mathbf{A}, w)=w$, when $w \in \mathbf{A}$.

Proof. $\Rightarrow$ : Suppose that for no $\alpha \in \mathcal{A}(w) w \in \mathbf{S}$ tries to $\alpha$. From Minimality, it follows that, for all $\alpha \in \mathcal{A}(w), w \neq f(\mathbf{S}$ tries to $\alpha, w)$. We now construct a counterexample to Success. We let $\llbracket p \rrbracket=\{w\}$. Then $\llbracket p \rrbracket^{w}=1$. But since for all for all $\alpha \in \mathcal{A}(w), f(\mathbf{S}$ tries to $\alpha, w) \notin\{w\}, \llbracket$ Can $p \rrbracket^{w}=0$.

$\Leftarrow$ : Suppose $w \in \mathbf{S}$ tries to $\alpha$, for some $\alpha \in \mathcal{A}(w)$. Now suppose $\llbracket \phi \rrbracket^{w}=1$. From Strong Centering, we can see that $f(\mathbf{S}$ tries to $\alpha, w)=w$. Since $w \in \llbracket \phi \rrbracket$, it follows that $\llbracket$ Can $\phi \rrbracket^{w}=1$.

Again, the reader can easily adapt the above proof to show the same condition characterises Can't-entails-won't.

\section{B Proofs of Main Results for $\mathcal{W}$ and Can}

Our language $\mathcal{L}$ is the set of sentences generated by the following grammar: 


$$
\phi::=p|\neg \phi| \phi \vee \psi|\mathcal{W} \phi| \operatorname{can} \phi|P A S T \phi| \diamond \phi \mid \nabla \phi
$$

Say that $\phi$ is non-modal iff it contains no instances of $\mathcal{W}$ or 'can'.

$\llbracket \cdot \rrbracket$, our semantic evaluation function, takes a (determinate) world $w$, a time $t$, a modal base $f$ and an indeterminate world (i.e. a set of worlds) $\mathcal{I}$ to a truth-value. Here are the relevant semantic entries:
a. $\quad \llbracket \neg \phi \rrbracket^{w, t, f, \mathcal{I}}=1$ iff $\llbracket \phi \rrbracket^{w, t, f, \mathcal{I}}=0$
b. $\quad \llbracket \neg \phi \rrbracket^{w, t, f, \mathcal{I}}=0$ iff $\llbracket \phi \rrbracket^{w, t, f, \mathcal{I}}=1$.
c. $\llbracket \neg \phi \rrbracket^{w, t, f, \mathcal{I}}=\#$ iff $\llbracket \phi \rrbracket^{w, t, f, \mathcal{I}}=\#$.

$\llbracket \phi \vee \psi \rrbracket^{w, t, f, \mathcal{I}}=$

a. $\quad 1$, if either $\llbracket \phi \rrbracket^{w, t, f, \mathcal{I}}=1$ or $\llbracket \psi \rrbracket^{w, t, f, \mathcal{I}}=1$

b. $\quad 0$, if $\llbracket \phi \rrbracket^{w, t, f, \mathcal{I}}=\llbracket \psi \rrbracket^{w, t, f, \mathcal{I}}=0$

c. \#, otherwise

a. $\llbracket \mathcal{W} \phi \rrbracket^{w, t, f, \mathcal{I}}$ is determinate only if either

(i) $s(\mathcal{I}, f(w, t)) \subseteq \llbracket \phi \rrbracket^{t, f, \mathcal{I}}$ or

(ii) $\quad s(\mathcal{I}, f(w, t)) \subseteq \llbracket \neg \phi \rrbracket^{t, f, \mathcal{I}}$

b. If determinate, $\llbracket \mathcal{W} \phi \rrbracket^{w, t, f, \mathcal{I}}=1$ iff $s(\mathcal{I}, f(w, t)) \subseteq \llbracket \phi \rrbracket^{t, f, \mathcal{I}}$

a. $\quad \llbracket \operatorname{can} \phi \rrbracket^{w, t, f, \mathcal{I}}$ is determinate only if either

(i) there is some $\alpha \in \mathcal{A}(w, t)$ such that $\llbracket \mathcal{W} \phi \rrbracket^{w, t, f^{\alpha}, \mathcal{I}}=1$;

(ii) or for all $\alpha \in \mathcal{A}(w, t), \llbracket \mathcal{W} \phi \rrbracket^{w, t, f^{\alpha}, \mathcal{I}^{2}}=0$.

b. If determinate, $\llbracket \operatorname{can} \phi \rrbracket^{w, t, f, \mathcal{I}}=1$ iff for some $\alpha \in \mathcal{A}(w, t): \llbracket \mathcal{W} \phi \rrbracket^{t, f^{\alpha}, \mathcal{I},}=$ 1

i.e. iff for some $\alpha \in \mathcal{A}(w, t): s(\mathcal{I}, f(w, t) \cap \alpha) \subseteq \llbracket \phi \rrbracket^{t, f^{\alpha}, \mathcal{I}}$

(74) a. $\quad \llbracket P A S T \phi \rrbracket^{w, t, f, \mathcal{I}}$ is determinate only if either

(i) there is some $t^{\prime}<t$ such that $\llbracket \phi \rrbracket^{w, t^{\prime}, \mathcal{I}}=1$

(ii) for all $t^{\prime}<t, \llbracket \phi \rrbracket^{w, t^{\prime}, f, \mathcal{I}}=0$

b. If determinate, $\llbracket P A S T \phi \rrbracket^{w, t, f, \mathcal{I}}=1$ iff for some $t^{\prime}<t: \llbracket \phi \rrbracket^{w, t^{\prime}, f, \mathcal{I}}=1$

a. $\quad \llbracket \diamond \phi \rrbracket^{w, t, f, \mathcal{I}}$ is determinate only if either

(i) there is some $w^{\prime} \in C(w, t)$, the circumstantially accessible worlds at $w$ and $t$, such that $\llbracket \phi \rrbracket^{w^{\prime}, t, f, \mathcal{I}}=1$

(ii) for all $w^{\prime} \in C(w, t) \llbracket \phi \rrbracket^{w^{\prime}, t, f, \mathcal{I}}=0$

b. If determinate, $\llbracket \diamond \phi \rrbracket^{w, t, f, \overline{\mathcal{I}}}=1$ iff for some $w^{\prime} \in C(w, t): \llbracket \phi \rrbracket^{w^{\prime}, t, f, \mathcal{I}}=$ 1

$$
\llbracket \nabla \phi \rrbracket^{w, t, f, \mathcal{I}}=1, \text { if } \llbracket \phi \rrbracket^{w, t, f, \mathcal{I}}=\# ;
$$


$\llbracket \nabla \phi \rrbracket^{w, t, f, \mathcal{I}}=0$, otherwise.

Note that from these assumptions we can derive the following entry for 'cannot':

a. $\llbracket$ cannot $\phi \rrbracket^{w, t, f, \mathcal{I}}$ is determinate only if either

(i) there is some $\alpha \in \mathcal{A}(w, t)$ such that $\llbracket \mathcal{W} \phi \rrbracket^{w, t, f^{\alpha}, \mathcal{I}}=1$;

(ii) or for all $\alpha \in \mathcal{A}(w, t), \llbracket \mathcal{W} \phi \rrbracket^{w, t, f^{\alpha}, \mathcal{I}, \mathcal{A}}=0$.

b. If determinate, $\llbracket \operatorname{cannot} \phi \rrbracket^{w, t, f, \mathcal{I}}=1$ iff for all $\alpha \in \mathcal{A}(w, t): \llbracket \mathcal{W} \phi \rrbracket^{t, f^{\alpha}, \mathcal{I}} \neq$ 1

i.e. iff for all $\alpha \in \mathcal{A}(w, t): s(\mathcal{I}, f(w, t) \cap \alpha) \nsubseteq \llbracket \phi \rrbracket^{t, f^{\alpha}, \mathcal{I}}$

i.e., given the determinacy conditions, iff for all $\alpha \in \mathcal{A}(w, t): s(\mathcal{I}, f(w, t) \cap$ $\alpha) \subseteq \llbracket \neg \phi \rrbracket^{t, f^{\alpha}, \mathcal{I}}$

Here are the various semantic assumptions I make:

Partitionality. $\mathcal{A}(w, t)$ is a partition of $C(w, t)$, the circumstantially accessible worlds at $w$ and $t$.

Circumstantial. $C(w, t)=\left\{w^{\prime}:\right.$ for all non-modal $\phi$, if $\left.t^{\prime}<t, \llbracket \phi \rrbracket^{w, t^{\prime}, f, \mathcal{I}}=\llbracket \phi \rrbracket^{w^{\prime}, t^{\prime}, f, \mathcal{I}}\right\}$

Overlap. If $\mathbf{A} \cap \mathbf{B} \neq \varnothing$, then $s(\mathbf{A}, \mathbf{B})=\mathbf{A} \cap \mathbf{B}$.

I assume the usual Kaplanian notion of truth at a context:

Truth at a context. $\llbracket \phi \rrbracket^{c}=\llbracket \phi \rrbracket^{w_{c}, t_{c}, f_{c}, \mathcal{I}_{c}}$

I make the following assumptions about the postsemantics:

Modal Bases. $f_{c}(w, t)=W$

Unsettled World. $\mathcal{I}_{c}=C\left(w_{c}, t_{c}\right)$

Action Time. If $t<t_{c}$, then if $\alpha \in \mathcal{A}\left(w_{c}, t\right)$ and $w_{c} \in \alpha$ then $\mathcal{I}_{c} \subseteq \alpha$.

This last assumption ensures that if an action available in the past was performed, it is now settled that it was performed. I assume the standard Kaplanian story for the postsemantics of the remaining indices.

Finally, our notions of validity:

Validity. $\phi_{1}, \ldots, \phi_{n} \models \psi$ iff whenever $\llbracket \phi_{1} \rrbracket^{w, t, f, \mathcal{I}}=\ldots=\llbracket \phi_{n} \rrbracket^{w, t, f, \mathcal{I}}=1$ then $\llbracket \psi \rrbracket^{w, t, f, \mathcal{I}}=1$. 
Diagonal validity. $\phi_{1}, \ldots, \phi_{n} \models_{\bar{c}} \psi$ iff whenever $\llbracket \phi_{1} \rrbracket^{c}=\ldots=\llbracket \phi_{n} \rrbracket^{c}=1$ then $\llbracket \psi \rrbracket^{c}=$ 1.

Strawson-validity. $\phi_{1}, \ldots, \phi_{n} \stackrel{\text { Str }}{=} \psi$ iff when $\llbracket \phi_{1} \rrbracket^{w, t, f, \mathcal{I}}=\ldots=\llbracket \phi_{n} \rrbracket^{w, t, f, \mathcal{I}}=1$ and $\llbracket \psi \rrbracket^{w, t, f, \mathcal{I}}$ is determinate, then $\llbracket \psi \rrbracket^{w, t, f, \mathcal{I}}=1$.

Diagonal Strawson-validity. $\phi_{1}, \ldots, \phi_{n} \frac{\text { Str }}{\bar{c}} \psi$ iff when $\llbracket \phi_{1} \rrbracket^{c}=\ldots=\llbracket \phi_{n} \rrbracket^{c}=1$ and $\llbracket \psi \rrbracket^{c}$ is determinate, then $\llbracket \psi \rrbracket^{c}=1$.

We now prove that the various results in the text:

Fact 1. $\diamond \phi, \diamond \neg \phi \mid \overline{\bar{c}} \nabla(\mathcal{W} \phi)$

Proof. Suppose $\llbracket \diamond \phi \rrbracket^{c}=\llbracket \diamond \neg \phi \rrbracket^{c}=1$. Then $C\left(w_{c}, t_{c}\right) \nsubseteq \llbracket \phi \rrbracket^{t_{c}, f_{c}, \mathcal{I}_{c}}$ and $C\left(w_{c}, t_{c}\right) \nsubseteq$ $\llbracket \neg \phi \rrbracket^{t_{c}, f_{c}, \mathcal{I}_{c}}$. By Unsettled World, $\mathcal{I}_{c}=C\left(w_{c}, t_{c}\right)$, so $\mathcal{I}_{c} \nsubseteq \llbracket \phi \rrbracket^{t_{c}, f_{c}, \mathcal{I}_{c}}$ and $\mathcal{I}_{c} \nsubseteq$ $\llbracket \neg \phi \rrbracket^{t_{c}, f_{c}, \mathcal{I}_{c}}$. By Overlap and Modal Bases, $s\left(\mathcal{I}_{c}, f_{c}\left(w_{c}, t_{c}\right)\right)=\mathcal{I}_{c}$. Since then $s\left(\mathcal{I}_{c}, f_{c}\left(w_{c}, t_{c}\right)\right) \nsubseteq \llbracket \phi \rrbracket^{t_{c}, f_{c}, \mathcal{I}_{c}}$ and $s\left(\mathcal{I}_{c}, f_{c}\left(w_{c}, t_{c}\right)\right) \nsubseteq \llbracket \neg \phi \rrbracket^{t_{c}, f_{c}, \mathcal{I}_{c}}, \llbracket \mathcal{W} \phi \rrbracket^{w_{c}, t_{c}, f_{c}, \mathcal{I}_{c}}=$ $\#$; so $\llbracket \nabla(\mathcal{W} \phi) \rrbracket^{w_{c}, t_{c}, f_{c}, \mathcal{I}_{c}}=1$

Fact 2. Where $\phi$ is non-modal, $P A S T \phi \bar{c}_{\bar{c}} P A S T(\mathcal{W} \phi)$

Proof. Suppose $\llbracket P A S T \phi \rrbracket^{c}=1$. Then there's some $t^{\prime}$ earlier than $t_{c}$ such that $\llbracket \phi \rrbracket^{w_{c}, t^{\prime}, f_{c}, \mathcal{I}_{c}}=1$. By Circumstantial and Unsettled World, it follows that $\mathcal{I}_{c} \subseteq$ $\llbracket \phi \rrbracket^{t^{\prime}, f_{c}, \mathcal{I}_{c}}$. By Modal Bases and Overlap, we have $s\left(\mathcal{I}_{c}, f\left(w, t^{\prime}\right)\right)=\mathcal{I}_{c}$. So there is some $t^{\prime}<t_{c}$, such that $s\left(\mathcal{I}_{c}, f\left(w, t^{\prime}\right)\right) \subseteq \llbracket \phi \rrbracket^{t^{\prime}, f_{c}, \mathcal{I}_{c}}$. So $\llbracket P A S T(\mathcal{W} \phi) \rrbracket^{w_{c}, t_{c}, f_{c}, \mathcal{I}_{c}}=$ 1.

Fact 3. $\neg \mathcal{W} \phi \mid \overline{\bar{c}} \mathcal{W} \neg \phi$

Proof. Suppose $\llbracket \neg \mathcal{W} \phi \rrbracket^{c}=1$. Since $\llbracket \neg \mathcal{W} \phi \rrbracket^{c}=1$ is determinate, it must be that either i) $s\left(\mathcal{I}_{c}, f_{c}\left(w_{c}, t_{c}\right)\right) \subseteq \llbracket \phi \rrbracket^{t_{c}, f_{c}, \mathcal{I}_{c}}$ or ii) $s\left(\mathcal{I}_{c}, f_{c}\left(w_{c}, t_{c}\right)\right) \subseteq \llbracket \neg \phi \rrbracket^{t_{c}, f_{c}, \mathcal{I}_{c}}$. Case i) is inconsistent with our initial assumption, so case ii) holds. That suffices for $\llbracket \mathcal{W} \neg \phi \rrbracket^{c}=1$

Fact 4. $\phi \vee \psi \not \|_{c}$ can $\phi \vee \operatorname{can} \psi$.

Proof. Take $\phi$ and $\neg \phi$. Suppose:

1. $\llbracket \phi \rrbracket^{w_{c}, t_{c}, f_{c}, \mathcal{I}_{c}}=1$

2. $\mathcal{A}\left(w_{c}, t_{c}\right)=\{S$ tries to $\phi, S$ tries to $\neg \phi\}$ 
3. S tries to $\phi$ and $S$ tries to $\neg \phi$ are consistent with both $\llbracket \phi \rrbracket^{t_{c}, f_{c}, \mathcal{I}_{c}}$ and $\llbracket \neg \phi \rrbracket^{t_{c}, f_{c}, \mathcal{I}_{c}}$

By $1, \llbracket \phi \vee \neg \phi \rrbracket^{w_{c}, t_{c}, f_{c}, \mathcal{I}_{c}}=1$. But the determinacy conditions for ${ }^{\ulcorner}$can $\left.\phi\right\urcorner$ are not met: for no $\alpha \in \mathcal{A}_{c}\left(w_{c}, t_{c}\right): \alpha \subseteq \llbracket \phi \rrbracket^{t_{c}, f_{c}, \mathcal{I}_{c}}$ or $\alpha \subseteq \llbracket \neg \phi \rrbracket^{t_{c}, f_{c}, \mathcal{I}_{c}}$. Similarly the determinacy conditions for ${ }^{r}$ can $\neg \phi$ ' are not met. So $\llbracket$ can $\phi \rrbracket^{w_{c}, t_{c}, f_{c}, \mathcal{I}_{c}}=\llbracket$ can $\neg \phi \rrbracket^{w_{c}, t_{c}, f_{c}, \mathcal{I}_{c}}=\#$. So $\phi \vee \psi \not \not_{c}$ can $\phi \vee$ can $\psi$.

Note that, since validity entails diagonal validity, we also have $\phi \vee \psi \not \models$ can $\phi \vee$ can $\psi$.

Fact 5. For non-modal $\phi, P A S T(\phi) \mid \bar{c} P A S T(\operatorname{can} \phi)$

Proof. Suppose $\llbracket P A S T \phi \rrbracket^{w_{c}, t_{c}, f_{c}, \mathcal{I}_{c}}=1$. By our entry for $P A S T$, there is some $t^{\prime}<t$ such that $\llbracket \phi \rrbracket^{w_{c}, t^{\prime}, f_{c}, \mathcal{I}_{c}}=1$. By Circumstantial, $C(w, t) \subseteq \llbracket \phi \rrbracket^{t^{\prime}, f_{c}, \mathcal{I}_{c}}$; and by Unsettled World, $\mathcal{I}_{c} \subseteq \llbracket \phi \rrbracket^{t^{\prime}, f_{c}, \mathcal{I}_{c}}$. Now by Partition, we know that there is some $\alpha \in \mathcal{A}_{c}\left(w_{c}, t^{\prime},\right)$ such that $w_{c} \in \alpha$. Action Time gives us that $\mathcal{I}_{c} \subseteq \alpha$.

Now we can show that $s\left(\mathcal{I}_{c}, f_{c}\left(w_{c}, t^{\prime}\right) \cap \alpha\right) \subseteq \llbracket \phi \rrbracket^{t^{\prime}, f_{c}, \mathcal{I}_{c}}$. By Modal Bases, $f_{c}\left(w_{c}, t^{\prime}\right) \cap \alpha=\alpha$. So $\mathcal{I}_{c} \subseteq f_{c}\left(w_{c}, t^{\prime}\right) \cap \alpha$. So, by Overlap, we have that $s\left(\mathcal{I}_{c}, f_{c}\left(w_{c}, t^{\prime}\right) \cap\right.$

$\alpha)=\mathcal{I}_{c}$. But we already know that $\mathcal{I}_{c} \subseteq \llbracket \phi \rrbracket^{t^{\prime}, f_{c}, \mathcal{I}_{c}}$. So $\exists \alpha \in \mathcal{A}_{c}\left(w_{c}, t^{\prime}\right): s\left(\mathcal{I}_{c}, f_{c}\left(w_{c}, t^{\prime}\right) \cap\right.$

$\alpha) \subseteq \llbracket \phi \rrbracket^{t^{\prime}, f_{c}, \mathcal{I}_{c}}$. Since $\phi$ is non-modal, $\llbracket \phi \rrbracket^{t^{\prime}, f_{c}, \mathcal{I}_{c}}=\llbracket \phi \rrbracket^{t^{\prime}, f_{c}^{\alpha}, \mathcal{I}_{c}}$; and so for some $t^{\prime}<t_{c}, \llbracket$ can $\phi \rrbracket^{w_{c}, t^{\prime}, f_{c}, \mathcal{I}_{c}}=1$. So $\llbracket P A S T(\operatorname{can} \phi) \rrbracket^{c}=1$.

Fact 6. When $\phi$ is non-modal, cannot $\phi \mid \overline{\bar{c}} \neg \phi$

Proof. Suppose $\llbracket$ cannot $\phi \rrbracket^{w_{c}, t_{c}, f_{c}, \mathcal{I}_{c}}=1$. Since it is determinate, either i) there is some $\alpha \in \mathcal{A}_{c}\left(w_{c}, t_{c}\right)$ such that $\llbracket \mathcal{W} \phi \rrbracket^{w_{c}, t_{c}, f_{c}^{\alpha}, \mathcal{I}_{c}}=1$; or ii) for all $\alpha \in \mathcal{A}_{c}\left(w_{c}, t_{c}\right)$, $\llbracket \mathcal{W} \phi \rrbracket^{w_{c}, t_{c}, f_{c}^{\alpha}, \mathcal{I}_{c}}=0$. Since $\llbracket$ cannot $\phi \rrbracket^{w_{c}, t_{c}, f_{c}, \mathcal{I}_{c}}=1$, i) cannot hold. ii) and the determinacy conditions for $\mathcal{W}$ gives us that for all $\alpha \in \mathcal{A}_{c}\left(w_{c}, t_{c}\right), \llbracket \mathcal{W} \neg \phi \rrbracket^{w_{c}, t_{c}, f_{c}+\alpha, \mathcal{I}_{c}}=$ 1. In other words, for all $\alpha \in \mathcal{A}_{c}\left(w_{c}, t_{c}\right), s\left(\mathcal{I}_{c}, f_{c}\left(w_{c}, t_{c}\right) \cap \alpha\right) \subseteq \llbracket \neg \phi \rrbracket^{t_{c}, f_{c}, \mathcal{I}_{c}}$.

By Partitionality, Circumstantial and Unsettled World, there's some $\alpha \in \mathcal{A}_{c}\left(w_{c}, t_{c}\right)$ such that $w_{c} \in \alpha$. This, together with Modal Bases, ensures $w_{c} \in f^{\alpha}\left(w_{c}, t_{c}\right)$. By Circumstantial and Unsettled World, we know $w_{c} \in \mathcal{I}_{c}$. By Overlap, we then know $w_{c} \in s\left(\mathcal{I}_{c}, f_{c}^{\alpha}\left(w_{c}, t_{c}\right)\right)$. But then, since $\forall \alpha \in \mathcal{A}_{c}\left(w_{c}, t_{c}\right), s\left(\mathcal{I}_{c}, f_{c}\left(w_{c}, t_{c}\right) \cap\right.$ $\alpha) \subseteq \llbracket \phi \rrbracket^{t_{c}, f_{c}^{\alpha}, \mathcal{I}_{c}}, w_{c} \in \llbracket \phi \rrbracket^{t_{c}, f_{c}^{\alpha}, \mathcal{I}_{c}}$, i.e. $\llbracket \phi \rrbracket^{w_{c}, t_{c}, f_{c}^{\alpha}, \mathcal{I}_{c}}=1$. When $\phi$ is non-modal, $\llbracket \phi \rrbracket^{w_{c}, t_{c}, f_{c}^{\alpha}, \mathcal{I}_{c}}=1$ iff $\llbracket \phi \rrbracket^{w_{c}, t_{c}, f_{c}, \mathcal{I}_{c}}=1$. So $\llbracket \phi \rrbracket^{w_{c}, t_{c}, f_{c}, \mathcal{I}_{c}}=1$.

Fact 7. Where $\phi$ is non-modal, $\phi \frac{\text { Str }}{\bar{c}}$ can $\phi$

Proof. Suppose $\llbracket \phi \rrbracket^{w_{c}, t_{c}, f_{c}, \mathcal{I}_{c}}=1$ and $\llbracket$ can $\phi \rrbracket^{w_{c}, t_{c}, f_{c}, \mathcal{I}_{c}}$ is determinate. If $\llbracket$ can $\phi \rrbracket^{w_{c}, t_{c}, f_{c}, \mathcal{I}_{c}}$ is determinate, then either i) there is some $\alpha \in \mathcal{A}_{c}\left(w_{c}, t_{c}\right)$ such that $\llbracket \phi \rrbracket^{w_{c}, t_{c}, f_{c}^{\alpha}, \mathcal{I}_{c}}=1$; or ii) for all $\alpha \in \mathcal{A}_{c}\left(w_{c}, t_{c}\right), \llbracket \phi \rrbracket^{w_{c}, t_{c}, f_{c}^{\alpha}, \mathcal{I}_{c}}=0$. By Partitionality, we know that for some $\alpha, w_{c} \in \alpha$. By reasoning analogous to that at the end of 
the proof of Fact 6, if $w_{c} \in \alpha, \phi$ is non-modal, and $\llbracket \mathcal{W} \phi \rrbracket^{w_{c}, t_{c}, f_{c}^{\alpha}, \mathcal{I}_{c}}=0$, then $\llbracket \phi \rrbracket^{w_{c}, t_{c}, f_{c}, \mathcal{I}_{c}}=0$. Since by supposition $\llbracket \phi \rrbracket^{w_{c}, t_{c}, f_{c}, \mathcal{I}_{c}}=1$, ii) cannot hold. So i) must hold. But i) entails $\llbracket$ can $\phi \rrbracket^{w_{c}, t_{c}, f_{c}, \mathcal{I}_{c}}=1$

\section{References}

Abusch, D. (1997). Sequence of tense and temporal de re. Linguistics and Philosophy, 20, 1-50.

Abusch, D. (1998). Generalizing tense semantics for future contexts. In S. Rothstein (Ed.) Events and Grammar (pp. 13-33). Dordrecht: Springer.

Austin, J. L. (1961). Ifs and Cans. In Philosophical Papers, (pp. 151-180). Oxford, UK: Oxford University Press.

Barnes, E. and Cameron, R. (2011). Back to the open future. Philosophical Perspectives, 25, 1-26.

Belnap, N., Perloff, M., and Xu, M. (2001). Facing the Future: Agents and Choices in Our Indeterminist World. Oxford, UK: Oxford University Press.

Bhatt, R. (1999). Ability modals and their actuality entailments. In K. Shahin, S. Blake, and E.S Kim (Eds.), WCCFL 17 Proceedings, Volume 17 (pp. 74-87). Stanford: CSLI Publications.

Bledin, J. (2014). Logic informed. Mind, 123, 277-316.

Brown, M. A. (1988). On the logic of ability. Journal of Philosophical Logic, 17, $1-26$.

Cariani, F. (2013). 'Ought' and Resolution Semantics. Nous, 47, 534-558.

Cariani, F. (2021). The modal future hypothesis debugged. Ms, University of Maryland.

Cariani, F. and Santorio, P. (2018). Will done better: Selection semantics, future credence, and indeterminacy. Mind, 127,129-165.

Ciardelli, I., Groenendijk, J., and Roelofsen, F. (2018). Inquisitive Semantics. Oxford: Oxford University Press.

Cinque, G. (1999). Adverbs and functional heads: A cross-linguistic perspective. Oxford: Oxford University Press. 
Condoravdi, C. (2002). Temporal interpretations of modals. In D. Beaver, S. Kaufman, B. Clark, and L. Casillas (Eds.), The Construction of Meaning (pp. 59-88). Stanford: CSLI Publications.

Copley, B. (2009). The Semantics of the Future. New York: Routledge.

Cross, C. B. (1986). 'Can' and the Logic of Ability. Philosophical Studies, 50, 53-64.

Fine, K. (2017). A theory of truthmaker content I: Conjunction, disjunction and negation. Journal of Philosophical Logic, 46, 625-674.

von Fintel, K. (1997). Bare plurals, bare conditionals, and only. Journal of Semantics, 14, 1-56.

von Fintel, K. (1999). NPI licensing, Strawson entailment, and context dependency. Journal of Semantics, 16(2):97-148.

Fodor, J. D. (1970). The Linguistic Description of Opaque Contexts. PhD thesis, MIT.

Fusco, M. (2021). Agential free choice. Journal of Philosophical Logic, 50, 57-87.

Goldstein, S. (2019). Free choice and homogeneity. Semantics and Pragmatics, 12.

Hacquard, V. (2006). Aspects of Modality. PhD thesis, MIT.

Hedden, B. (2012). Options and the subjective ought. Philosophical Studies, 158, 343-360.

Hedden, B. (2015). Reasons Without Persons: Rationality, Identity, and Time. Oxford: Oxford University Press.

Hilpinen, R. (1969). An Analysis of Relativised Modalities. In J. Davis, D. Hockney, and W. Wilson (Eds.), Philosophical Logic, Volume 20 (pp. 181 - 191). Dordrecht: Springer.

Horty, J. F. (2001). Agency and Deontic Logic. Oxford, UK: Oxford University Press.

Horty, J. F. and Belnap, N. (1995). The deliberative stit: A study of action, omission, ability, and obligation. Journal of Philosophical Logic, 24, 583-644.

Humberstone, I. L. (1981). From worlds to possibilities. Journal of Philosophical Logic, 10, 313-339. 
Kaufmann, S. (2005). Conditional truth and future reference. Journal of Semantics, $22,231-280$.

Kenny, A. (1976). Human Abilities and Dynamic Modalities. In J. Manninen, and R. Tuomela (Eds.), Essays on Explanation and Understanding (pp. 209-232). Dordrecht: Springer.

Kratzer, A. (1977). What 'Must' and 'Can' Must and Can Mean. Linguistics and Philosophy, 1, 337-355.

Kratzer, A. (1981). The Notional Category of Modality. In H. Eikmeyer, and H. Rieser (Eds.), Words, Worlds, and Contexts: New Approaches in Word Semantics (pp. 38-74). Berlin: de Gruyter.

Kratzer, A. (1991). Modality. In A. von Stechow, and D. Wunderlich (Eds.), Semantics: An International Handbook of Contemporary Research (pp. 639-650). Berlin: de Gruyter.

Kratzer, A. (2012). Conditionals. In Modals and Conditionals (pp.86-108). Oxford, UK: Oxford University Press.

Kriz, M. (2015). Aspects of Homogeneity in the Semantics of Natural Language. $\mathrm{PhD}$ thesis, University of Vienna.

Lehrer, K. (1968). Cans without ifs. Analysis, 29, 29-32.

Lehrer, K. (1976). 'Can' in Theory and Practice: A Possible Worlds Analysis. In M. Brand and D. Walton (Eds.), Action Theory (pp. 241-270). Dordrecht: Springer.

Lewis, D. (1976). The Paradoxes of Time Travel. American Philosophical Quarterly, 13, 145-152.

Lukasiewicz, J. (1920). On three-valued logic. Ruch filozoficzny, 5, 170-171.

Lukasiewicz, J. (1951). Aristotle's Syllogistic From the Standpoint of Modern Formal Logic. Oxford, UK: Clarendon Press.

MacFarlane, J. (2003). Future contingents and relative truth. Philosophical Quarterly, 53, 321-336.

Maier, J. (2018). Ability, modality, and genericity. Philosophical Studies, 175, 411-428.

Mandelkern, M., Schultheis, G., and Boylan, D. (2017). Agentive modals. The Philosophical Review, 126, 301-343. 
Ninan, D. (2021). Assertion, evidence, and the future. Ms, Tufts University.

Prior, A. (1967). Past, Present and Future. Oxford, UK: Clarendon Press.

Santorio, P. (2018). Alternatives and truthmakers in conditional semantics. The Journal of Philosophy, 115, 513-549.

Schwarzschild, R. (1993). Plurals, presuppositions and the sources of distributivity. Natural Language Semantics, 2, 201-248.

Stalnaker, R. (1968). A Theory of Conditionals. In N. Rescher (Ed.), Studies in Logical Theory (pp. 98-112). Oxford, UK: Blackwell.

Thomason, R. H. (1970). Indeterminist time and truth-value gaps. Theoria, 36, 264-281.

Thomason, R. H. (1984). Combinations of tense and modality. In D. Gabbay, and F. Guenthner (Eds.), Handbook of Philosophical Logic: Extensions of Classical Logic. Dordrecht: Springer.

Thomason, R. H. (2005). Ability, action, and context. MS, University of Michigan.

Veltman, F. (1985). Logics for Conditionals. PhD thesis, University of Amsterdam.

Willer, M. (2021). Two puzzles about ability can. Linguistics and Philosophy, forthcoming.

Williamson, T. (2000). Knowledge and its Limits. Oxford, UK: Oxford University Press.

Yalcin, S. (2007). Epistemic modals. Mind, 116, 983 - 1026. 